\title{
Analysis of deubiquitinase OTUD5 as a biomarker and therapeutic target for cervical cancer by bioinformatic analysis
}

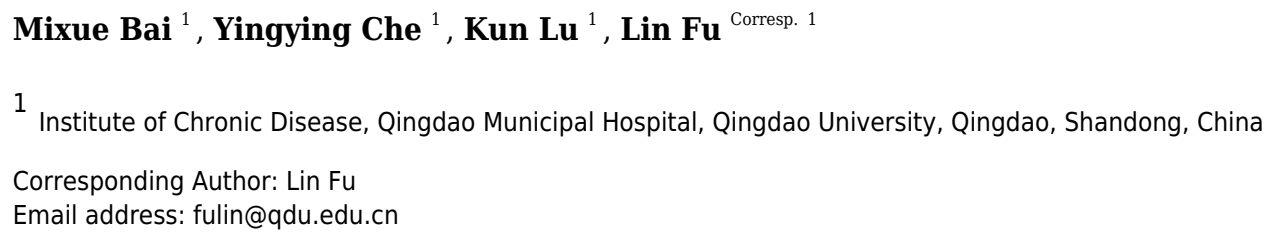

OTU deubiquitinase 5 (OTUD5), as a member of the ovarian tumor protease (OTU) family, was previously reported to play important roles in DNA repair and immunity. However, little is known about its function in tumors. Cervical cancer is a malignant tumor that seriously endangers the lives of women. Here, we found that low expression of OTUD5 in cervical cancer is associated with poor prognosis. Its expression is associated with tumor stage, metastatic nodes and tumor subtypes such as those related to the phosphatidylinositol-3-kinase (PI3K) - AKT signaling, epithelial-mesenchymal transition (EMT) and hormones. In addtion, we analyzed the coexpressed genes, related miRNAs, transcription factors, kinases, E3s and interacting proteins of OTUD5. We demonstrated that OTUD5 affects the expression levels of WD repeat domain 45 (WDR45), ubiquitinspecific peptidase 11 (USP11), GRIP1 associated protein 1 (GRIPAP1) and RNA binding motif protein 10 (RBM10). Moreover, hsa-mir-137, hsa-mir-1913, hsa-mir-937, hsa-mir-607, hsa-mir-3149 and hsa-mir-144 may inhibit the expression of OTUD5. Furthermore, we performed enrichment analysis of 22 coexpressed genes, 33 related miRNAs and 30 interacting proteins. In addition to ubiquitination and immunology related processes, they also participate in Hippo signaling, insulin signaling, EMT, histone methylation and phosphorylation kinase binding. Our study for the first time analyzed the expression of OTUD5 in cervical cancer and its relationship with clinicopathology and provided new insights for further study of its regulatory mechanism in tumors. 
1

2

3

4

5

6

7

8

9

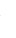

\section{Analysis of deubiquitinases OTUD5 as a biomarker and therapeutic target for} cervical cancer by bioinformatic analysis

Mixue Bai ${ }^{1}$, Yingying $\mathrm{Che}^{1}$, Kun $\mathrm{Lu}^{1}$, Lin $\mathrm{Fu}^{1 *}$

1. Institute of Chronic Disease, Qingdao Municipal Hospital, Qingdao University, Qingdao 266000, China.

*Corresponding authors: Dr. Lin Fu, E-mail: fulin@qdu.edu.cn 


\section{Abstract}

OTU deubiquitinase 5 (OTUD5), as a member of the ovarian tumor protease (OTU) family, was previously reported to play important roles in DNA repair and immunity. However, little is known about its function in tumors. Cervical cancer is a malignant tumor that seriously endangers the lives of women. Here, we found that low expression of OTUD5 in cervical cancer is associated with poor prognosis. Low expression of OTUD5 is associated with tumor stage, metastatic nodes and tumor subtypes such as those related to the phosphatidylinositol-3-kinase (PI3K) - AKT signaling, epithelial-mesenchymal transition (EMT) and hormones. In addtion, we analyzed the coexpressed genes, related miRNAs, transcription factors, kinases, E3s and interacting proteins of OTUD5. We demonstrated that OTUD5 affects the expression levels of WD repeat domain 45 (WDR45), ubiquitin-specific peptidase 11 (USP11), GRIP1 associated protein 1 (GRIPAP1) and RNA binding motif protein 10 (RBM10). Moreover, hsa-mir-137, hsa-mir-1913, hsa-mir-937, hsamir-607, hsa-mir-3149 and hsa-mir-144 may inhibit the expression of OTUD5. Furthermore, we performed enrichment analysis of 22 coexpressed genes, 33 related miRNAs and 30 interacting proteins. In addition to ubiquitination and immunology related processes, they also participate in Hippo signaling, insulin signaling, EMT, histone methylation and phosphorylation kinase binding. Our study, for the first time analyzed the expression of OTUD5 in cervical cancer and its relationship with clinical characteristics and provided new insights for further study of its regulatory mechanism in tumors.

Keywords: OTUD5, cervical cancer, bioinformatics, co-expression genes, protein-protein 


\section{interaction.}

\section{Introduction}

Cervical cancer is one of the most common malignancies among women worldwide and seriously affects women's quality of life ${ }^{1}$. Cervical cancer has a long pre-invasive phase that can be detected by clinical and histopathological examination. Human papillomavirus (HPV) has been identified as a pathogenic factor in the progression from pre-invasive to invasive cervical cancer, which is critical for the transformation of cervical epithelial cells ${ }^{2,3}$.

Ubiquitin is a small molecule protein composed of 76 amino acids that is widely present in all eukaryotic cells, and the sequence is highly conserved. There are only 3 amino acids differences between yeast and humans. The full length contains 7 lysine sites (K6, K11, K27, K29, K33, K48, K63).

Ubiquitination is a dynamic post-translational modification in which target protein binds to ubiquitin molecules through an enzymatic reaction. The main function of ubiquitination is to participate in the degradation of target proteins and the clearance of abnormal proteins. The synergy of three ubiquitinated enzymes is usually required: E1 ubiquitin-activating enzymes, E2 ubiquitin-conjugating enzymes, and E3 ubiquitin-ligase enzymes.

Ubiquitination, which can affect the homeostasis of the body, is an important post-translational modification $(\mathrm{PTM})^{4}$. Ubiquitination disorders also play important roles in diseases such as cancer $^{5}$, neurodegenerative disease ${ }^{6}$, osteoporosis ${ }^{7}$, muscular dystrophy ${ }^{8}$ and immune disease ${ }^{9}$. Ubiquitination is a reversible physiological process. Deubiquitinases (DUBs) remove ubiquitin molecules from its substrate, balancing the regulation of ubiquitin modification balanced. DUB 
activity can affect cell homeostasis, protein stability, and a variety of signaling pathways.

Ubiquitination is a reversible mechanism by which deubiquitinases (DUBs) remove ubiquitin from their substrates DUBs consist of six families, including the ubiquitin-specific proteases (USPs), ovarian tumor proteases (OTUs), ubiquitin C-terminal hydrolases (UCHs), Machado-Joseph disease proteins (MJDs), the Jab1/MPN/Mov34 metalloenzymes (JAMMs) and motif interacting with UB-containing novel DUB (MINDYs) subfamily ${ }^{10,11}$. OTU deubiquitinase 5 (OTUD5), also known as DUBA, is an important DUB in the OTU family ${ }^{12}$. Substrates that have been discovered so far are: Ku80, p53, PDCD5, STP16, UBR5 and TRAF3. OTUD5 plays an important role in DNA repair and immunity. In response to genotoxic stress, OTUD5 interacts with p53-PDCD5 to reduce its ubiquitination ${ }^{13,14}$. OTUD5 interacts with Ku80 to positively regulate non-homologous end joining NHEJ-mediated repair ${ }^{15}$. In addition, OTUD5 stabilizes SPT16 and regulates SPT16 dependent Pol II elongation ${ }^{16}$. In immunomodulation, TGF- $\beta$ stimulation leads to excessive OTUD5 production. Then, OTUD5 stabilizes UBR5, causing ubiquitination of ROR $\gamma \mathrm{t}$ in mobilized T cells ${ }^{17}$. As a negative regulator of innate immunity, OTUD5 removes the k63 chain ubiquitination of TRAF3, ultimately suppressing IFN-I ${ }^{18}$. However, the function of OTUD5 in tumors is not clear.

In the present study, we found that reduced expression of OTUD5 is correlated with poor prognosis in cervical cancer. Moreover, we also analyzed the miRNAs and transcription factors that may regulate the expression of OTUD5, as well as kinases and E3s that may mediate the posttranslational regulation of OTUD5. Finally, through the enrichment analysis of coexpressed genes, miRNAs and interacting proteins, we discovered new processes and pathways related to 
OTUD5.

\section{Methods}

\subsection{Cells culture and transfection}

HeLa cells (obtained from the American Type Culture Collection) were maintained in DMEM supplemented with $10 \%$ fetal bovine serum and $1 \%$ penicillin/streptomycin in a humidified atmosphere with $5 \% \mathrm{CO}_{2}$ at $37{ }^{\circ} \mathrm{C}$. For the knockdown assay, cells were transfected with appropriate siRNAs against OTUD5 using Lipofectamine 2000, and scrambled siRNA was used as a control. After $48 \mathrm{~h}$, the cells were collected, and the efficiency of OTUD5 knockdown was verified by qRT-PCR.

\subsection{RNA interference}

HeLa cells were transfected with siRNAs for OTUD5 knockdown. The sequences of the siRNA against OTUD5 are as follows: \#1: 5'-GGGCUGGGCCUGCCAU

CAUUC-3', \#2: 5'-GGGCCCUCAUUCAGCAGAUGU-3'.

\subsection{GEPIA}

GEPIA (Interactive Analysis of Gene Expression Analysis, http://gepia.cancer-pku.cn/), ${ }^{19}$ a webbased tool that provides fast and customizable functionality based on TCGA and GTEx data. GEPIA provides key interactive and customizable features, including gene expression, correlation analysis, and patient survival analysis. We used GEPIA to analyze the expression of OTUD5, and its relationship with transcription factors, kinases in CESC.

\subsection{Oncomine analysis}

DNA copy numbers of OTUD5 in CESC were determined from in the Oncomine 4.5 database. 
100 Oncomine (www.oncomine.org) ${ }^{20}$ contains 715 gene expression data sets and data from 86,733

101 cancer tissues and normal tissues. The analysis of OTUD5 is based on CESC studies of Scotto

102 Cervix. We investigated the HPV infection status of CESC patients whether HPV status correlated with the copy number of OTUD5.

\subsection{UALCAN analysis}

UALCAN is an interactive web portal in-depth using RNA-seq and clinical data from 31 cancer

types in TCGA ${ }^{21}$. One of the feature is that it allows analysis of the relative expression of query genes in tumors and normal samples, as well as in individual tumor subgroups, based on individual cancer stages, tumor grades, or other clinicopathological features. UALCAN is publicly available from http://ualcan.path.uab.edu. We analyzed the relationship between OTUD5 expression and clinical characteristics including CESC patient age, race, weight, lymph node metastasis, and tumor stage using UALCAN. In addition, we also analyzed the relationship between methylation and OTUD5 in CESC. In addition, the correlation OTUD5 and ELK1 in CESC was assessed via UALCAN.

\subsection{LinkedOmics analysis}

The LinkedOmics database (http://www.LinkedOmics.org/login.php) is a web-based platform for analyzing 32 cancers datasets from the TCGA ${ }^{22}$. LinkedOmics' LinkFinder module was used to study the differentially expressed genes associated with OTUD5 in the TCGA CESC cohort $(\mathrm{n}=$ 307). Genes coexpressed with OTUD5 in CESC were statistically analyzed using Pearson correlation coefficients. All results are graphically displayed on the volcano and heat map. The 
121

122

123

124

125

126

127

128

129

130

131

132

133

134

135

136

137

138

139

140

141

OTUD5. FDR $<0.05$ was set as the threshold.

\subsection{GeneMANIA analysis}

GeneMANIA (http://www.genemania.org) is a user-friendly and convenient web interface for building protein-protein interaction (PPI) networks, generating hypotheses about gene function, analyzing gene lists and prioritizing them. Functional analysis ${ }^{23}$. The site can set the source of the network edge and has several bioinformatics methods: physical interaction with OTUD5. We visualized the physical interaction gene network using GeneMANIA.

\section{8 cBioPortal analysis}

The cBioPortal for Cancer Genomics (http://cbioportal.org) is an open-source tool for interactive exploration of multidimensional cancer genomics datasets and currently contains 308 cancer studies ${ }^{24,25}$. We used cBioPortal to analyze OTUD5 alteration in TCGA-CESC. Search parameters included mutations and copy number alterations.

\subsection{GO analysis and KEGG analysis}

We performed GO analysis and KEGG analysis of coexpressed genes and interacting proteins of OTUD5 in the Metascape website (http://metascape.org). The GO and KEGG analysis of miRNA was performed with DIANA tools (http:/www.microrna.gr/miRPathv3/). The threshold was set mini genes $>3, \mathrm{P}<0.05$.

\subsection{The Kaplan-Meier plotter}

The prognostic value of the expression of miRNAs, including hsa-mir-137, hsa-mir-1913, hsamir-937, hsa-mir-607, hsa-mir-3149 and hsa-mir-144, was evaluated using the online database Kaplan-Meier Plotter (www.kmplot.com). To analyze the overall survival (OS) of patients with 
142 CESC, patient samples were split into two groups by median expression (high vs. low expression)

143 and assessed by a Kaplan-Meier survival plot, and the results included hazard ratios (HRs) with

$14495 \%$ confidence intervals (CI) and log-rank p-values.

$145 \quad 2.11$ qRT-PCR

146 Total RNA was isolated using TRIzol reagent (Invitrogen, Carlsbad, CA). First strand cDNA was 147 synthesized subsequently using the Fast Quant RT Kit (with gDNase) (TOYOBO, Japan) 148 according to the product manual. Quantitative polymerase chain reaction (qPCR) was performed 149 in three replicate wells on an ABI 7500 Real-Time PCR System (Thermo Fisher Scientific, 150 Waltham, MA) using SuperReal PreMix Plus (SYBR Green) (Tiangen Biotech, Beijing, China).

GAPDH, forward: CATGAGAAGTATGACAACAGCCT;

AGTCCTTCCACGATACAAAG. WDR45, forward: GTGGTAGTAGTCCCAA

GTTCTC; reverse: CGATCACGATCTTGTCATGG. TFE3: forward: AAGGAACGGCA

GAAGAAAGAC; reverse: CACTGGACTTAGGGATGAGAG. TBC1D25, forward:

GCCCTTTACACAGTCCATCC; reverse: TGAAACTCAGCATCGCTCAG. RBM10, forward:

TCCCAGTATTACTACAATGCTCAG; reverse: CTTCTCCTTCTTCTCTTTGC

CC. USP11, forward: CCGTGACTACAACAACTCCT; reverse: TCGTCATCTTCTTTC

TCATCCC. GRIPAP1, forward: CAGTAGCATCTCCTCCTTCAG; reverse: CTCCTCC

AGCATCCATTTCTC. The relative expression levels of OTUD5, WDR45, TFE3, TBC1D25,

USP11, RBM10 and GRIPAP1 were calculated using the $2^{-\Delta \Delta C T}$ method and GAPDH was used as an internal control. Each experiment was repeated three times.

\section{12 Statistical analysis}


163 The cut-off values for OTUD5, USP11, RBM10, WDR45 and GRIPAP1 expression were

164 determined by their median values. All analyses, including the t-test and correlation analysis were

165 performed by GraphPad Prism. In our analysis, P values less than 0.05 were considered significant.

1663 Results

167 3.1 OTUD5 is expressed at abnormally low levels in CESC with poor prognosis

168 To detect the role of OTUD5 in cervical cancer, we first analyzed the survival rate and OTUD5

expression in cervical cancer patients. Clinical survival data were derived from TCGA-CESC,

containing 13 control samples (expression-TMP = 59.59) and 306 CESC samples (expression-

TMP $=36.3)$. The samples were divided into a low OTUD5 expression group $(\mathrm{n}=98)$ and a high

OTUD5 expression group $(\mathrm{n}=193)$ according to the median (FIG. 1A). Kaplan-Meier survival

analysis revealed that low mRNA expression of OTUD5 was significantly correlated with poor

overall survival $(\mathrm{P}=0.0031)$. Then, we evaluated the expression level of OTUD5 in the TCGA

database (supplementary FIG.1A). The expression of OTUD5 in cervical cancer tissues was lower

than that in normal tissues (FIG. 1B). In addition, OTUD5 was significantly underexpressed in

cervical cancer cell lines(HT-3 and DoTc2-4510) according to COSMIC database analysis

(Supplementary FIG. 1B). To explore the underlying mechanisms, we first analyzed mutations in

OTUD5 in CESC. The missense mutation frequency of OTUD5 was approximately $1.57 \%$ (FIG.

1C). Mutations included P478Q (in endocervical adenocarcinoma) and Q199R and E241K (in

cervical squamous cell carcinoma). However, the current reports demonstrated that only mutations

in the S177A and C224S sites affect the deubiquitinase activity of OTUD5 ${ }^{15,26,27}$.For the copy

number variation, deep deletion samples $(n=2)$ and shallow deletion samples $(n=42)$ exist in 
184 CESC patients (FIG. 1D). Additionally, methylation affected OTUD5 expression in CESC (FIG.

185 1E). There was no meaningful difference in methylation levels between the tumor and normal

186 groups (FIG. 1F). OTUD5 copy number, mRNA and mutations all may affect its expression in

187 cervical cancer, and regulate the progression of tumors. Taken together, the low expression of

OTUD5 is associated with poor prognosis in cervical cancer and OTUD5 is a potential tumor

suppressor gene. In addition, OTUD5 undergoes mutational inactivation and loss of copy number

in cervical cancer tissues. Under this double attack, the ability OTUD5 to inhibit cervical cancer

191

192

193 is suppressed.

\subsection{The association of OTUD5 expression with clinicopathologic characteristics in cervical}

\section{cancer}

Next, we analyzed the relationship between OTUD5 and clinicopathological aspects. OTUD5 was negatively related to tumor stage and number of lymph nodes (FIG. 2A-B). The expression levels of OTUD5 showed no difference in groups classified by age, race, or weight (FIG. 2C-E). Since high-risk HPV infection is a key step in cervical carcinoma, we next analyzed the patients infected with HPV in the Oncomine-Scotto Cervix ${ }^{28}$. We found that the copy number of OTUD5 was not different in patients with or without HPV infection (FIG. 2F). We speculated that OTUD5 may be regulated after transcription, for example, by miRNA and PTM. Next, we analyzed the relationship of OTUD5 in subtypes associated with EMT, hormones and PI3K-AKT. Epithelial-mesenchymal transition (EMT) is one of the main causes of cancer metastasis ${ }^{29}$. EMT-activated transcription factors are invasive and motility characteristics for cervical cancer ${ }^{30}$. The expression level of OTUD5 was lower in EMT related subtypes (FIG. 2G). Hormones are not directly involved in 
205

206

207

208

209

210

211

212

213

214

215

216

217

218

219

220

221

222

223

224

225

metabolism, but can promote or inhibit the body's original metabolic processes by regulating

certain proteins. For example, oral contraceptives can affect the secretion of hormones and increase

the risk of cervical cancer ${ }^{31}$. OTUD5 is also expressed at a lower level in hormone-related

subtypes (FIG. 2H). In cervical cancer, activation of PI3K-AKT signaling is closely related to

tumor progression and poor prognosis ${ }^{30}$. Consistently, OTUD5 is also downregulated in PI3K-

AKT related subtypes (FIG. 2I).

Taken together, the mRNA levels of OTUD5 in cervical tumor tissues are negatively related to tumor stage and node metastasis. OTUD5 expressed at low levels in CESC subtypes associated with EMT, hormones and PI3K-AKT.

\subsection{Enrichment analysis of OTUD5 coexpressed genes in CESC}

To determine the potential function OTUD5 in CESC, we analyzed genes associated with OTUD5 in the LinkedOmics database. The RNA-seq data were obtained from 307 samples from TCGACESC. In the volcano plot, 3907 genes (dark red dots) displayed meaningful positive correlations with OTUD5, whereas 3660 genes (dark green dots) displayed meaningful negative correlations (FIG. 3A). The 50 significant gene sets positively related to OTUD5 are shown in the heat map (FIG. 3B). Among them, we selected 22 genes had have lower expression correlated with poor overall survival in CESC for further analysis (TABLE 1). The GO term analysis performed by using the Metascape database is shown in FIG. 3C and 3D. These genes were mainly involved in modification-dependent macromolecule catabolic processes

(GO:0019941), modification-dependent protein catabolic processes (GO:0043632), ubiquitin-dependent protein catabolic processes (GO:0006511), proteolysis involved in cellular protein catabolic processes 
(GO:0051603), cellular protein catabolic process (GO:0044257) and cellular component disassembly (GO:0022411) (FIG. 3C). The top 3 noteworthy terms of the GO functional analysis were modification-dependent protein binding (GO:0140030), methylated histone binding (GO:0035064) and histone binding (GO:0042393) (FIG. 3D).

Next, we analyzed the correlation between these coexpressed genes with OTUD5 in GEPIA (TABLE 1). Four genes USP11 (Pearson correlation $=0.72, \mathrm{P}=2.38 \mathrm{E}-23)$, RBM10 (Pearson correlation $=0.73, \mathrm{P}=5.27 \mathrm{E}-43)$, WDR45 $($ Pearson correlation $=0.76, \mathrm{P}=1.63 \mathrm{E}-57)$ and GRIPAP1 (Pearson correlation $=0.84, \mathrm{P}=2.09 \mathrm{E}-45$ ), had strong positive correlations with OTUD5 (FIG. 4A-D). USP11 is a member of the USP family protein that is involved in several cancers including cervical ${ }^{32}$, lung, liver, brain and colon cancer. RBM10 is a member of the RNAbinding protein (RBP) family and is known for its role in mRNA splicing. Preliminary works have shown that RBM10 can increase apoptosis and inhibit cell proliferation ${ }^{33,34}$. WDR45, which encodes a WD40 repeat-containing PtdIns(3)P binding protein, is crucial for the basic autophagy pathway $^{35}$. GRIPAP1 (GRIP-related protein 1) is a neuron-specific endosome protein that is identified as part of the AMPAR complex, as it directly interacts with AMPAR. GRIPAP1 has been reported to play an important role in promoting the maturation of recovered endosomes ${ }^{36}$. In addition, the low expression of these genes in CESC is related to poor prognosis in CESC (FIG. 4E-H). To clarify the relationship of these genes with OTUD5, we knocked down OTUD5 in HeLa cells and then performed qRT-PCR to detect their mRNA levels. We found that USP11, RBM10, WDR45 and GRIPAP1 were downregulated after knocking down OTUD5 (FIG. 4I-L). OTUD5 and its coexpressed genes may regulate the ubiquitination of proteins and affect the occurrence 
247 and development of cervical cancer.

248 3.4 The miRNAs and transcription factors that may regulate the mRNA expression of 249 OTUD5 in CESC.

250 To identify the upstream molecules that regulate the mRNA levels of OTUD5 in cervical cancer, 251 we analyzed miRNAs and transcription factors related to OTUD5. We first evaluated the miRNA252 OTUD5 network in CESC using Linked Omics. In the volcano plot, 475 miRNAs (dark red dots) displayed meaningful positive correlations with OTUD5, and 334 miRNAs (dark green dots) displayed significant negative correlations (FIG. 5A). Since miRNAs usually inhibit the expression of target genes, we are more interested in negatively related miRNAs. The 33 denoting miRNAs negatively correlated with OTUD5 are shown in the heat map (FIG. 5B).

We performed GO and KEGG analyses by searching the DIANA database (TABLE 2). These miRNAs were enriched in Hippo signaling (hsa04390), protein processing in the endoplasmic reticulum (hsa04141), viral carcinogenesis (hsa05203), fatty acid metabolism (hsa01212), adherens junctions (hsa04520) and proteoglycans in cancer (hsa05205) (FIG. 5C). These miRNAs were primarily involved in biological processes such as symbiosis, encompassing mutualism through parasitism (GO:0043903), cellular nitrogen compound metabolic processes (GO:0034641), cellular protein modification processes (GO:0006464), biosynthetic processes (GO:0009058), gene expression (GO:0010467) and small molecule metabolic processes (GO:0044281) (FIG. 5D). Their molecular functions were enriched in ion binding (GO:0043167), enzyme binding (GO:0019899), poly(A) RNA binding (GO:0008143), protein binding transcription factor activity (GO:0000988), RNA binding (GO:0003723), and cytoskeletal protein 
268

269

270

271

272

273

274

275

276

277

278

279

280

281

282

283

284

285

286

287

288

binding (GO:0008092) (FIG. 5E). They were predominantly localized at microtubule organizing center (GO:0005815), protein complex (GO:0031519), nucleoplasm (GO:0005654), focal adhesion (GO:0005925), cytosol (GO:0005829) and endosome (GO:0005768) (FIG. 5F). Among the top ten relevant miRNAs, we found that hsa-mir-137, hsa-mir-1913, hsa-mir-937, hsa-mir-607, hsa-mir-3149 and hsa-mir-144 were associated with poor overall survival in CESC (FIG. 5G-H and TABLE 2). Therefore, these miRNAs may influence the prognosis of cervical cancer by regulating the expression of OTUD5.

The transcription factors that regulate OTUD5 were previously unknown. Next, we predicted the transcription factors of OTUD5 using QIAGEN, ConTra v3 and PROMO. As shown in TABLE 3, there were 4 potential candidates from QIAGEN, 7 potential candidates from ConTra v3 and 9 potential candidates from PROMO. We also examined the correlation between these transcription factors and OTUD5. The expression level of OTUD5 was significantiy correlated with the transcription factors SP1, SP2, SP3, SP4, MAZ, MAZR, ELF4, IRF-2, YY1, TFIID, FOXP3, GTF2I, NF-1, ELK1 and P53 in CESC (TABLE 3). Therefore, we speculated that transcription factors may affect the expression of OTUD5 in cervical cancer.

\subsection{Protein-protein interaction network (PPI) and enrichment analysis of proteins}

\section{interacting with OTUD5}

As a DUB, OTUD5 always functions by binding to another protein and deubiquitinating it. Through proteome analysis, we found 30 interacting proteins of OTUD5 and displayed them in a physical interaction network using GeneMANIA (FIG 6. A). The five most reliable proteins were ARPC3, LONRF2, GPX4, LANCL2 and GYS1 (FIG6.A). 
289 Next, we performed GO enrichment analysis of these 30 interacting proteins. Since OTUD5 is a

290 DUB, it is not surprising that the interacting proteins are enriched in ubiquitin protein ligase

291 binding (GO:0031625) and ubiquitin-like protein ligase binding (GO:0044389) (FIG 6. B). In

292 addition, these proteins also have the function of binding GTPases (GO:0051020) and kinases

293 (GO:0019900) (FIG 6. B). Moreover, we noticed significant enrichment of functions corrected

294 with actin binding (GO:0003779) and actin filament binding (GO:0051015) and processes related

295 to positive regulation of organelle organization (GO:0006996), actin filament-based process

296 (GO:0030029) and actin cytoskeleton organization (GO:0030036) (FIG 6. B and C). This suggests

297 that OTUD5 may affect the formation of the cytoskeleton by interacting with these proteins.

298 OTUD5 has been found to interact with UBR5 and TRAF3 and regulate immune reactions ${ }^{17,18}$.

299 Consistent with this, these proteins are primarily involved in the regulation of immune

response-activating signal transduction (GO:0002757), activation of immune response

301 (GO:0002253), and immune response-regulating signaling pathway (GO:0002764) (FIG 6. C).

302 These proteins are mainly localized to the cell-cell junction (GO:0005911), cell cortex

303 (GO:0005938), focal adhesions (GO:0005925), cell-substrate adheres junctions (GO:0005924),

304 cell-substrate junctions (GO:0030055) and cytoplasmic regions (GO:0099568) (FIG 6. D). The numerous components of the cytoskeleton, actin and tubulin, are highly integrated and their functions are well coordinated in normal cells. In contrast, mutations and abnormal expression of

cytoskeletal and cytoskeleton-related proteins play an important role in the ability of cancer cells to resist chemotherapy and metastasis ${ }^{37,38}$. OTUD5 may regulate the occurrence or metastasis of cervical cancer through its interacting protein. Here, these analyses revealed that the localization 
310 of the above proteins on the cell membrane and may affect the information transfer between cells

311 by regulating cell signaling, such as apoptosis, WNT/ $\beta$-catenin, PI3K-AKT, p53, NF- $\mathrm{B}$ signaling

312 pathways and EMT. Signaling pathways affect the occurrence and development of multiple

313 tumors. KEGG analysis illustrated enrichment in the insulin signaling pathway (hsa04910),

314 indicated by enrichment of genes such as GRB2, GYS1 and PKLR. Consistent with this, Insulin-

315 like growth factor 1(IGF1R) is reported to promote cervical cancer development ${ }^{39}$.

316 Phosphorylation of OTUD5 at Ser177 is critical to the enzyme activity of OTUD5 ${ }^{26}$.in our results,

317 the most important of kinase-target networks related primarily to the kinases PLK1, ATM,

318 PIK3CA, CDK5 and CHEK1. Among them, PLK1, ATM, CDK5 and CHEK1 are abnormally

319 expressed in cervical cancer (TABLE 4). The predicted E3s capable of regulating OTUD5 are

320 shown in TABLE 5. The expression of NEDDL4, RNF6, MDM2 and TRIM11 is abnormal in

321 cervical cancer. In summary, we analyzed the interacting proteins of OTUD5 and predicted the

322 potential kinases and E3s of OTUD5.

\section{Discussion}

324 Increasing epidemiological data have shown that cervical cancer is the most popular malignancy

325 among women worldwide. The long-term survival rate of CESC patients is low due to the ease of

326 recurrence and metastasis. The occurrence and development of malignant tumors is usually a

327 multistage complex process, involving a variety of genes and signal transduction pathways. Many

328 genes, including BAX and p53 associated with Bcl-2, have been reported to play an important role

329 in the progression of cervical cancer ${ }^{40}$. Therefore, the study of abnormal CESC gene changes is

330 vital to further clarify the mechanisms of occurrence and development of cervical cancer. 
331 OTUD5 was previously reported to play important roles in DNA damage and immune regulation.

332 Little is known about its function in tumors. In the present study, bioinformatic analysis via high

333 throughput RNA-sequencing data from TCGA indicated that low OTUD5 expression in CESC

334 and was correlated with leading clinical features (tumor stage, nodal metastasis status) and overall

335 survival. This revealed that OTUD5 may act as a possible biomarker of prognosis and treatment

336 target in CESC.

337 Phosphorylation of Thr308 and Ser473 leads to the activation of PI3K-AKT signaling ${ }^{30}$. OTUD5 was reported to affect the phosphorylation of Akt in cervical tumor cells ${ }^{41}$. In this analysis, the expression level of OTUD5 was consistently downregulated in PI3K-AKT subtype tumors. The proteins interacting with OTUD5 were involved in the function of kinase binding.

In recent years, there have been increasing number of reports on the relationship between epigenetic modification and tumors ${ }^{27}$. The methylation of histones is closely related to tumors ${ }^{42}$.

Our analysis suggested that although OTUD5 is methylated in cervical cancer, its coexpressed genes may affect the progression of cervical cancer by regulating methylation.

In addition, HPV, as a virus, has been identified as a pathogenic factor in cervical cancer. The miRNAs related to OTUD5 are enriched in viral carcinogenesis. They may regulate cervical cancer caused by HPV infection by regulating OTUD5. The Hippo signaling pathway is reported to be active in cervical cancer (33). The functions of OTUD5 in the Hippo pathway are not clear. According to our results, miRNAs associated with OTUD5 are enriched in Hippo pathway. The new regulatory relationship between miRNA and OTUD5 may be involved in the regulatory network of the Hippo pathway in cervical cancer. 
352 In cervical cancer, the predicted kinases have been reported to perform different functions. For 353 example, the c-ABL-PLK1 axis is a new prognostic marker and treatment target in cervical cancers

$354{ }^{43}$. Osthole treatment also sensitized cervical cancer to irradiation, revealing promoted DNA 355 damage and prohibited ATM/NF-kB signaling ${ }^{44}$. CDK5 phosphorylates p53 at serine 20 and 356 serine 46 residues, thereby promoting its recruitment to $\mathrm{p} 21$ and BAX promoters in cervical cancer 357 45. CHEK1 and p-CHEK1 contribute to the development of cervical cancer ${ }^{46}$. This illustrates a 358 new model between phosphorylation and ubiquitination.

\section{5. Conclusions}

In summary, through bioinformatics analysis, we obtained a comprehensive view of the role of OTUD5 in cervical cancer. OTUD5 is a protein expressed at low levels and associated with poor prognosis. Analysis of OTUD5-related genes, miRNAs and transcription factors; proteins interacting with OTUD5; and kinase and E3s targeting OTUD5 and further analysis of GO and pathway enrichment and PPIs, may provide new regulatory mechanisms associated with OTUD5 in cervical cancer.

\section{Acknowledgments}

This project is supported by grants from the China Postdoctoral Science Foundation [2018M640612, 2019T120568] and the National Natural Science Foundation of China [81702743].

\section{Conflicts of interest}

There are no conflicts of interest to declare.

\section{References}


3731 Vu, M., Yu, J., Awolude, O. A. \& Chuang, L. Cervical cancer worldwide. Curr Probl Cancer 42, 457-465, 374 doi:10.1016/j.currproblcancer.2018.06.003 (2018).

3752 Goodman, A. HPV testing as a screen for cervical cancer. BMJ 350, h2372, doi:10.1136/bmj.h2372 (2015).

3763 Clifford, G. M., Tully, S. \& Franceschi, S. Carcinogenicity of Human Papillomavirus (HPV) Types in HIV-

377 Positive Women: A Meta-Analysis From HPV Infection to Cervical Cancer. Clinical Infectious Diseases 64, 378 1228-1235, doi:10.1093/cid/cix135 (2017).

3794 Swatek, K. N. \& Komander, D. Ubiquitin modifications. Cell Res 26, 399-422, doi:10.1038/cr.2016.39 $380 \quad$ (2016).

3815 Bang, S., Kaur, S. \& Kurokawa, M. Regulation of the p53 Family Proteins by the Ubiquitin Proteasomal Pathway. Int J Mol Sci 21, doi:10.3390/ijms21010261 (2019).

6 Boland, B. et al. Promoting the clearance of neurotoxic proteins in neurodegenerative disorders of ageing. Nat Rev Drug Discov 17, 660-688, doi:10.1038/nrd.2018.109 (2018).

7 Fukushima, H. et al. NOTCH2 Hajdu-Cheney Mutations Escape SCF(FBW7)-Dependent Proteolysis to Promote Osteoporosis. Mol Cell 68, 645-658.e645, doi:10.1016/j.molcel.2017.10.018 (2017).

Lazzari, E. \& Meroni, G. TRIM32 ubiquitin E3 ligase, one enzyme for several pathologies: From muscular dystrophy to tumours. The international journal of biochemistry \& cell biology 79, 469-477, doi:10.1016/j.biocel.2016.07.023 (2016).

Lopez-Castejon, G. Control of the inflammasome by the ubiquitin system. The FEBS journal 287, 11-26, doi:10.1111/febs.15118 (2020).

401

Nijman, S. M. et al. A genomic and functional inventory of deubiquitinating enzymes. Cell 123, 773-786, doi:10.1016/j.cell.2005.11.007 (2005). Important for Genome Stability. Mol Cell 70, 150-164.e156, doi:10.1016/j.molcel.2018.02.023 (2018).

2 Mevissen, T. E. et al. OTU deubiquitinases reveal mechanisms of linkage specificity and enable ubiquitin chain restriction analysis. Cell 154, 169-184, doi:10.1016/j.cell.2013.05.046 (2013).

13 Judong Luo1. OTUD5 Regulates p53 Stability by Deubiquitinating p53 doi:10.1371/

10.1371/journal.pone.0077682.g001 (2013).

4 Park, S. Y. et al. Deubiquitinase OTUD5 mediates the sequential activation of PDCD5 and p53 in response to genotoxic stress. Cancer Lett 357, 419-427, doi:10.1016/j.canlet.2014.12.005 (2015).

5 Li, F. et al. The deubiquitinase OTUD5 regulates Ku80 stability and non-homologous end joining. Cell Mol Life Sci, doi:10.1007/s00018-019-03094-5 (2019).

6 de Vivo, A. et al. The OTUD5-UBR5 complex regulates FACT-mediated transcription at damaged chromatin. Nucleic Acids Res 47, 729-746, doi:10.1093/nar/gky1219 (2019).

7 Rutz, S. et al. Deubiquitinase DUBA is a post-translational brake on interleukin-17 production in T cells. Nature 518, 417-421, doi:10.1038/nature13979 (2015).

8 Kayagaki, N. et al. DUBA: a deubiquitinase that regulates type I interferon production. Science 318, 16281632, doi:10.1126/science.1145918 (2007).

9 Tang, Z. et al. GEPIA: a web server for cancer and normal gene expression profiling and interactive analyses. Nucleic Acids Res 45, W98-W102, doi:10.1093/nar/gkx247 (2017).

20 Rhodes, D. R. et al. Oncomine 3.0: genes, pathways, and networks in a collection of 18,000 cancer gene expression profiles. Neoplasia 9, 166-180, doi:10.1593/neo.07112 (2007). 
41421 Chandrashekar, D. S. et al. UALCAN: A Portal for Facilitating Tumor Subgroup Gene Expression and Survival Analyses. Neoplasia 19, 649-658, doi:10.1016/j.neo.2017.05.002 (2017).

Vasaikar, S. V., Straub, P., Wang, J. \& Zhang, B. LinkedOmics: analyzing multi-omics data within and across 32 cancer types. Nucleic Acids Res 46, D956-D963, doi:10.1093/nar/gkx1090 (2018).

Warde-Farley, D. et al. The GeneMANIA prediction server: biological network integration for gene prioritization and predicting gene function. Nucleic Acids Res 38, W214-220, doi:10.1093/nar/gkq537 (2010).

4 Jianjiong gao, B. a. a., 1 Ugur Dogrusoz,2 gideon Dresdner,1 Benjamin gross, 1, S. Onur Sumer, Y. S., 1 anders Jacobsen,1 Rileen Sinha,1 erik larsson,3 ethan cerami,1,4 \& chris Sander, n. S. integrative analysis of complex cancer genomics and clinical Profiles Using the cBioPortal. doi:10.1126/scisignal.2004088] (2008). Cerami, E. et al. The cBio cancer genomics portal: an open platform for exploring multidimensional cancer genomics data. Cancer Discov 2, 401-404, doi:10.1158/2159-8290.CD-12-0095 (2012).

Huang, O. W. et al. Phosphorylation-dependent activity of the deubiquitinase DUBA. Nat Struct Mol Biol 19, 171-175, doi:10.1038/nsmb.2206 (2012).

$\mathrm{Wu}$, J. et al. Systematical identification of cell-specificity of CTCF-gene binding based on epigenetic modifications. Briefings in bioinformatics, doi:10.1093/bib/bbaa004 (2020).

Scotto, L. et al. Integrative genomics analysis of chromosome $5 \mathrm{p}$ gain in cervical cancer reveals target overexpressed genes, including Drosha. Mol Cancer 7, 58, doi:10.1186/1476-4598-7-58 (2008).

Qureshi, R., Arora, H. \& Rizvi, M. A. EMT in cervical cancer: its role in tumour progression and response to therapy. Cancer Lett 356, 321-331, doi:10.1016/j.canlet.2014.09.021 (2015).

Jiang, C. et al. p53R2 overexpression in cervical cancer promotes AKT signaling and EMT, and is correlated with tumor progression, metastasis and poor prognosis. Cell Cycle 16, 1673-1682, doi:10.1080/15384101.2017.1320629 (2017).

1 Smith, J. S. et al. Cervical cancer and use of hormonal contraceptives: a systematic review. The Lancet 361, 1159-1167, doi:10.1016/s0140-6736(03)12949-2 (2003).

32 Lin, C. H., Chang, H. S. \& Yu, W. C. USP11 stabilizes HPV-16E7 and further modulates the E7 biological activity. J Biol Chem 283, 15681-15688, doi:10.1074/jbc.M708278200 (2008).

3 Zhao, J. et al. Functional analysis reveals that RBM10 mutations contribute to lung adenocarcinoma pathogenesis by deregulating splicing. Sci Rep 7, 40488, doi:10.1038/srep40488 (2017).

34 Bechara, E. G., Sebestyen, E., Bernardis, I., Eyras, E. \& Valcarcel, J. RBM5, 6, and 10 differentially regulate NUMB alternative splicing to control cancer cell proliferation. Mol Cell 52, 720-733, doi:10.1016/j.molcel.2013.11.010 (2013).

5 Zhao, Y. G. et al. The autophagy gene Wdr45/Wipi4 regulates learning and memory function and axonal homeostasis. Autophagy 11, 881-890, doi:10.1080/15548627.2015.1047127 (2015).

Hoogenraad, C. C. et al. Neuron specific Rab4 effector GRASP-1 coordinates membrane specialization and maturation of recycling endosomes. PLoS biology 8, e1000283, doi:10.1371/journal.pbio.1000283 (2010).

Fife, C. M., McCarroll, J. A. \& Kavallaris, M. Movers and shakers: cell cytoskeleton in cancer metastasis. British journal of pharmacology 171, 5507-5523, doi:10.1111/bph.12704 (2014).

8 Hall, A. The cytoskeleton and cancer. Cancer metastasis reviews 28, 5-14, doi:10.1007/s10555-008-9166-3 (2009).

39 Steller, M. A., Delgado, C. H., Bartels, C. J., Woodworth, C. D. \& Zou, Z. Overexpression of the insulin-like 
455

456

457

458

459

460

461

462

463

$464 \quad 43$

465

$466 \quad 44$

467

$468 \quad 45$

469

470

$471 \quad 46$

472

473 growth factor-1 receptor and autocrine stimulation in human cervical cancer cells. Cancer Res 56, 1761-1765 (1996).

40 Karlidag, T. et al. Expression of Bax, p53, and p27/kip in patients with papillary thyroid carcinoma with or without cervical nodal metastasis. Am J Otolaryngol 28, 31-36, doi:10.1016/j.amjoto.2006.06.008 (2007).

41 Yin, F. et al. Effect of Deubiquitinase Ovarian Tumor Domain-Containing Protein 5 (OTUD5) on Radiosensitivity of Cervical Cancer by Regulating the Ubiquitination of Akt and its Mechanism. Med Sci Monit 25, 3469-3475, doi:10.12659/MSM.912904 (2019).

42 Audia, J. E. \& Campbell, R. M. Histone Modifications and Cancer. Cold Spring Harb Perspect Biol 8, a019521, doi:10.1101/cshperspect.a019521 (2016).

43 Xu Yang1, Gang Chen2,7, Wei Li3,6,7, Changmin Peng4, Yue Zhu1, Xiaoming Yang1, Teng Li5*, Cheng Cao3* and Huadong Pei1*. Cervical cancer growth is regulated by a c-ABL-PLK1 signaling axis. (2017).

44 Che, Y. et al. Osthole enhances antitumor activity and irradiation sensitivity of cervical cancer cells by suppressing ATM/NFkappaB signaling. Oncol Rep 40, 737-747, doi:10.3892/or.2018.6514 (2018).

45 Ajay, A. K. et al. Cdk5 phosphorylates non-genotoxically overexpressed p53 following inhibition of PP2A to induce cell cycle arrest/apoptosis and inhibits tumor progression. Mol Cancer 9, 204, doi:10.1186/14764598-9-204 (2010).

46 Mazumder Indra, D. et al. Inactivation of CHEK1 and EI24 is associated with the development of invasive cervical carcinoma: clinical and prognostic implications. Int J Cancer 129, 1859-1871, doi:10.1002/ijc.25849 (2011). 
Figure Legend

A

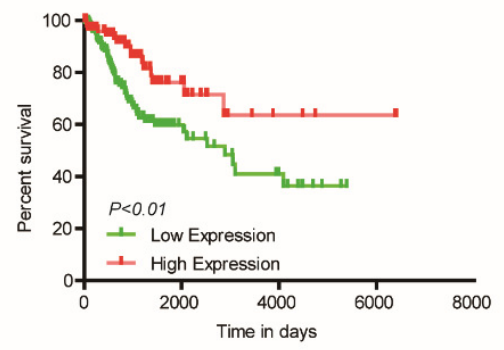

B
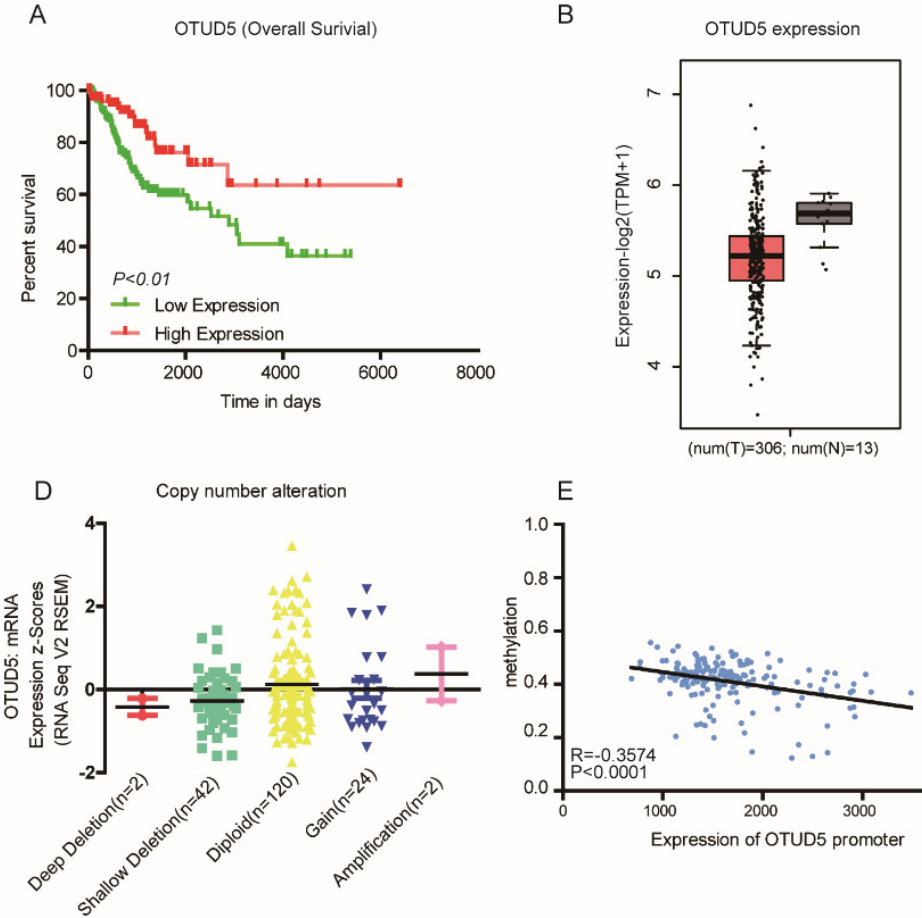

E

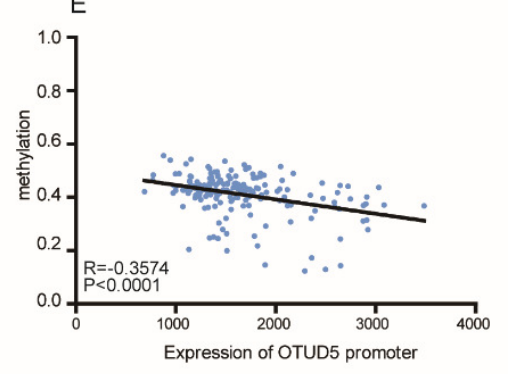

C

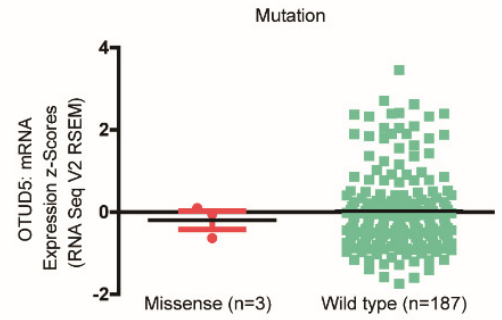

$\mathrm{F}$

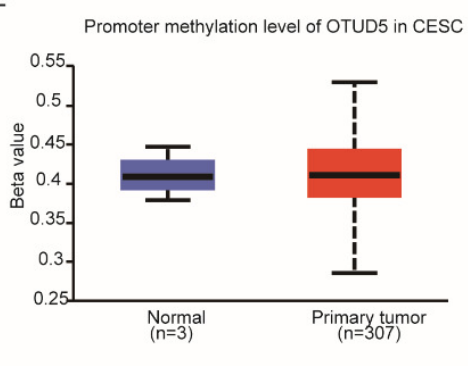

FIG. 1. The expression of OTUD5 in CESC.

(A) Overall survival of CESC patients (TCGA). (B) Box plot showing the transcripts per million (TPM) of OTUD5 in CESC (TCGA) from the GEPIA database. (C-D) Scatter plot showing mutation and copy number alteration of OTUD5 in CESC patients using cBioPortal. (E) The correlation of OTUD5 and methylation in CESC data from MethHC (http://methhc.mbc.nctu.edu.tw/php/index.php). (F) Box plot showing OTUD5 methylation in CESC via UALCAN. The beta value indicates the level of DNA methylation ranging from 0 (unmethylated) to 1 (fully methylated). 


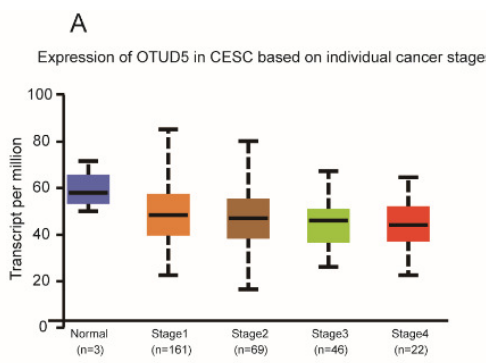

D

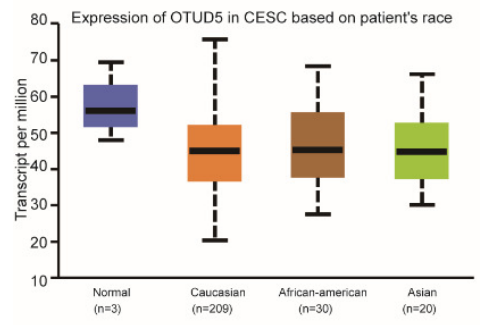

G

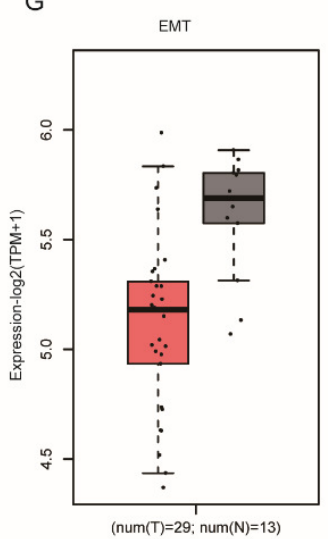

B

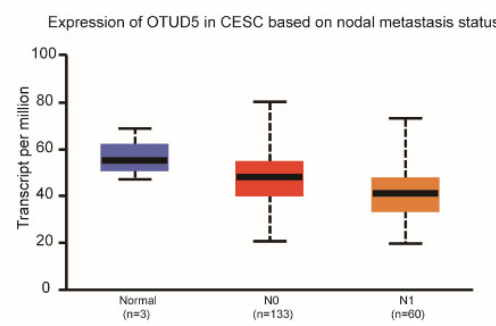

E

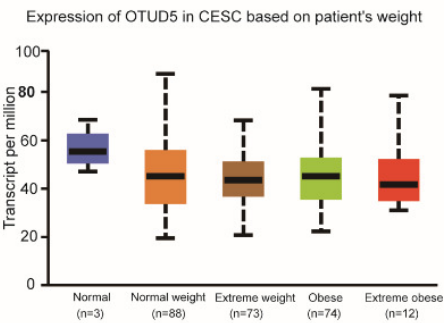

$\mathrm{H}$

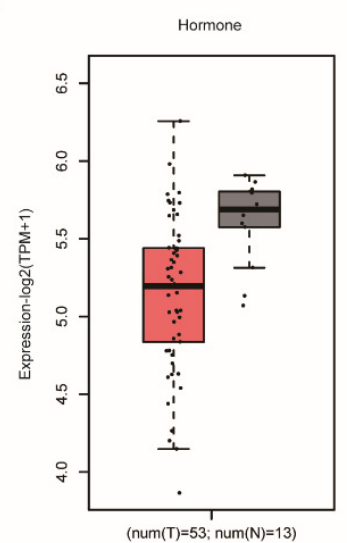

C

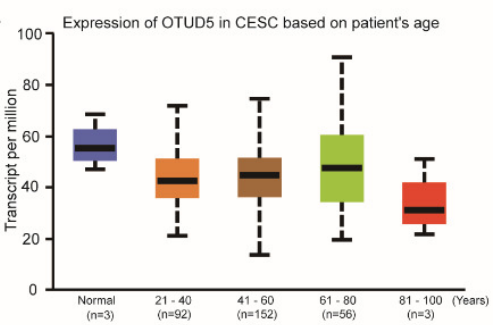

F
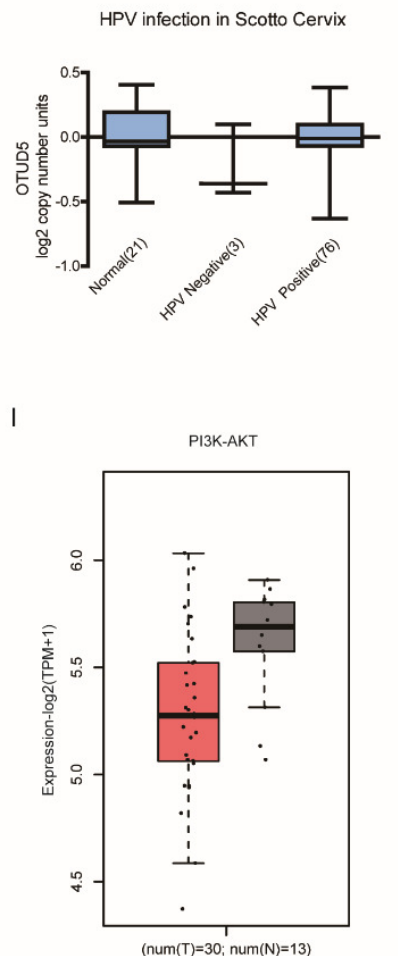

487

488

489

490

491

492

493

494

495

FIG. 2. The relationship between OTUD5 expression and the clinical pathology of cervical cancer.

(A)Boxplot showing the relative expression of OTUD5 by tumor stage (stage 1, stage 2, stage 3, stage 4 or stage 5) in CESC patients via UALCAN. (B) Boxplot showing relative expression of OTUD5 by nodal metastasis status (normal, N0 or N1) from normal controls and CESC patients via UALCAN. (C-E)Boxplot showing the relative expression of OTUD5 by age (aged 21-40, 41$60,61-80$, or 81-100 years), weight (normal, or extreme obese) and race (normal or Asian) from CESC patients via UALCAN. (F) Box plot showing OTUD5 copy number in the TCGA Scotto Cervix dataset via ONCOMINE. (G) Box plot showing the effect of OTUD5 on epithelial- 
496 mesenchymal Transition (EMT) in CESC patients in GEPIA database. (H) Box plot showing the 497 effect of OTUD5 on hormone in CESC patients in the GEPIA database. (I) Box plot showing the 498 effect of OTUD5 on the PI3K-AKT pathway in CESC patients in the GEPIA database.

499

500

501

502

503

504

505

506

507

508

509

510

511

512

513

514 

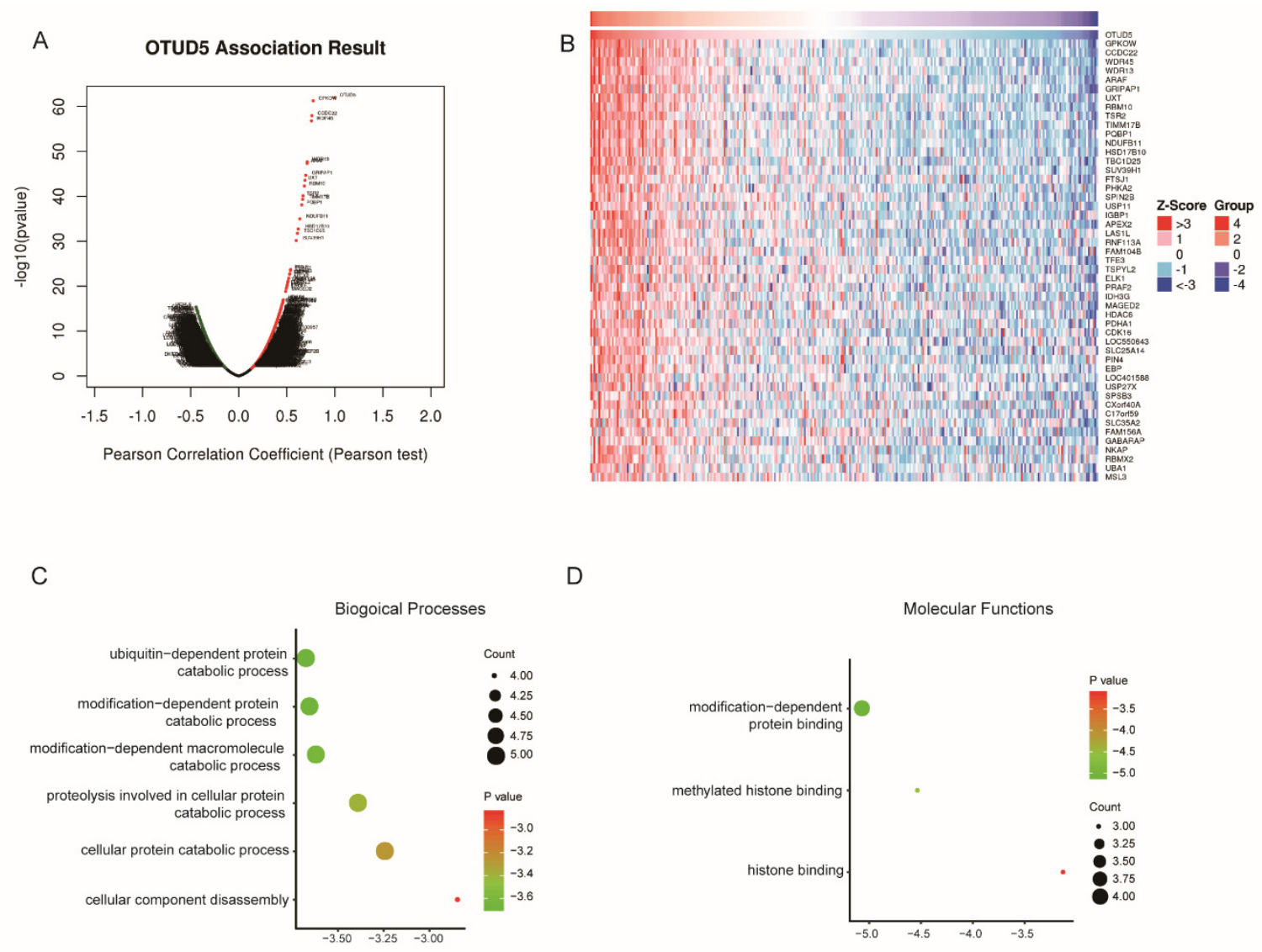

FIG 3. GO Functional Annotation and Pathway Enrichment of OTUD5 Coexpressed Genes in TCGA-CESC.

518 (A)A Pearson test was used to analyze correlations between OTUD5, and genes differentially 519 expressed in CESC. (B) Heat maps showing genes positively correlated with OTUD5 in CESC 520 (TOP 50). (C-D) Enrichment analysis of the genes altered in the OTUD5 coexpression genes in cervical cancer. The box plot displays the enrichment results of the top 22 genes altered in the OTUD5 coexpression genes in CESC. (C) Biological processes. (D) Molecular functions. 

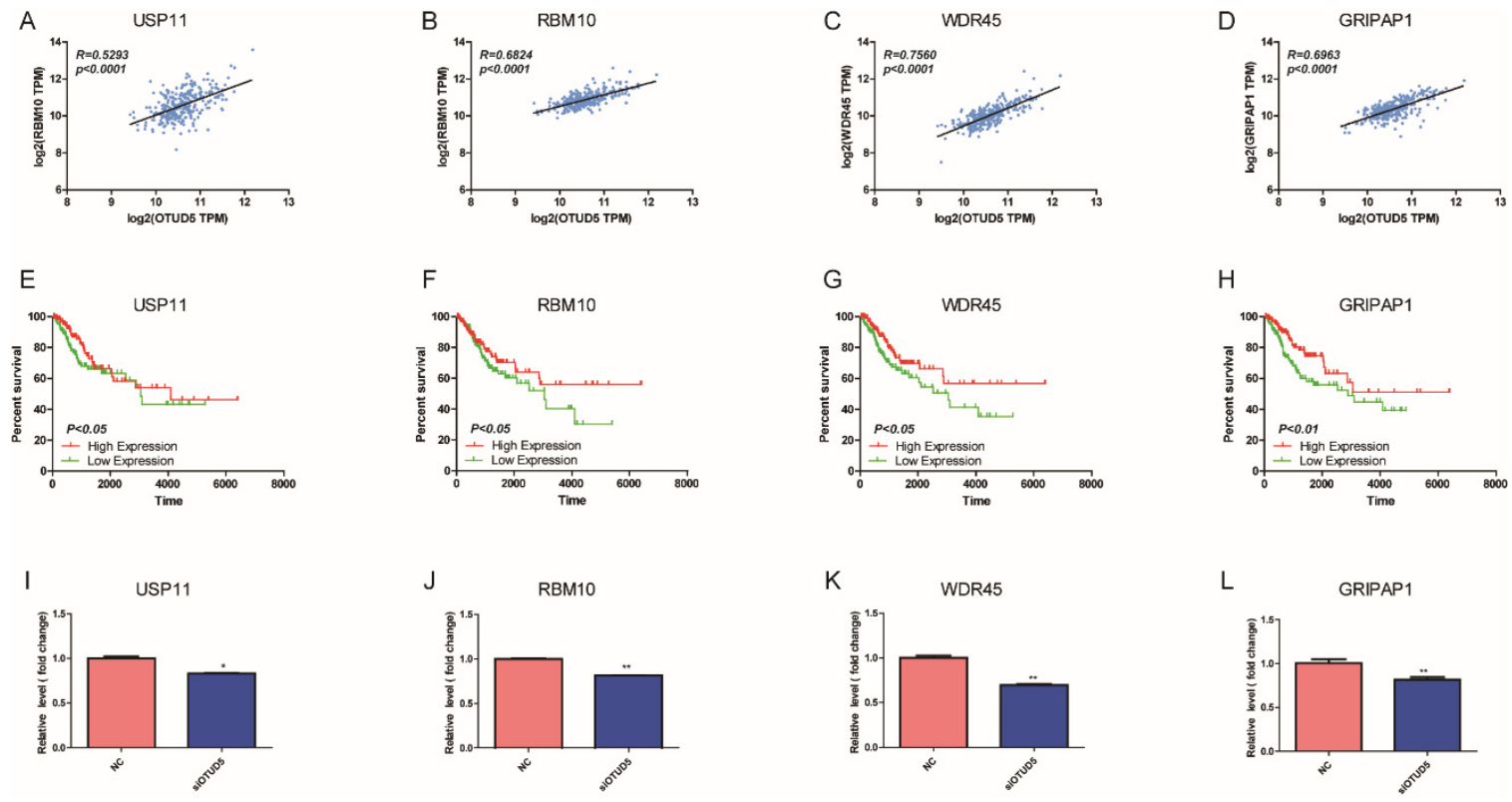

FIG 4. USP11, RBM10, WDR45 and GRIPAP1 are associated with OTUD5.

(A-D) The scatter plot shows the Pearson correlation of OTUD5 expression with USP11, RBM10,

WDR45 and GRIPAP1 expression in CESC patients (LinkedOmics). (E-H) Overall survival curve showing USP11, RBM10, WDR45 and GRIPAP1 in CESC patients (linkedomics), low expression ( $n=137)$, and high expression $(n=136)$. (I-L) mRNA levels of USP11, RBM10, WDR45 and GRIPAP1 were detected by qRT-PCR when OTUD5 was knocked down in HeLa cells using siRNA. 
A

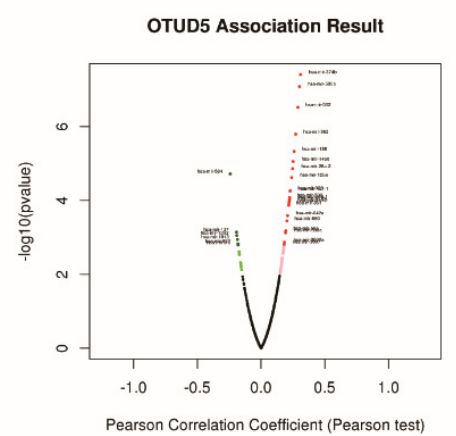

C

Pathway

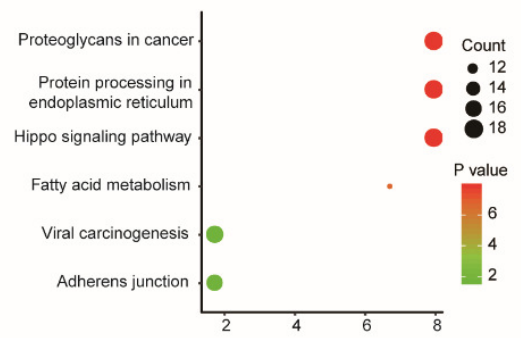

F

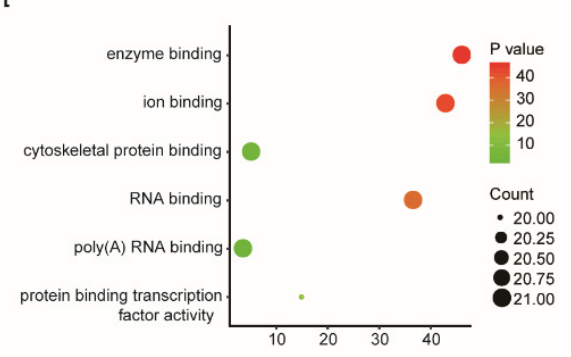

$\mathrm{H}$
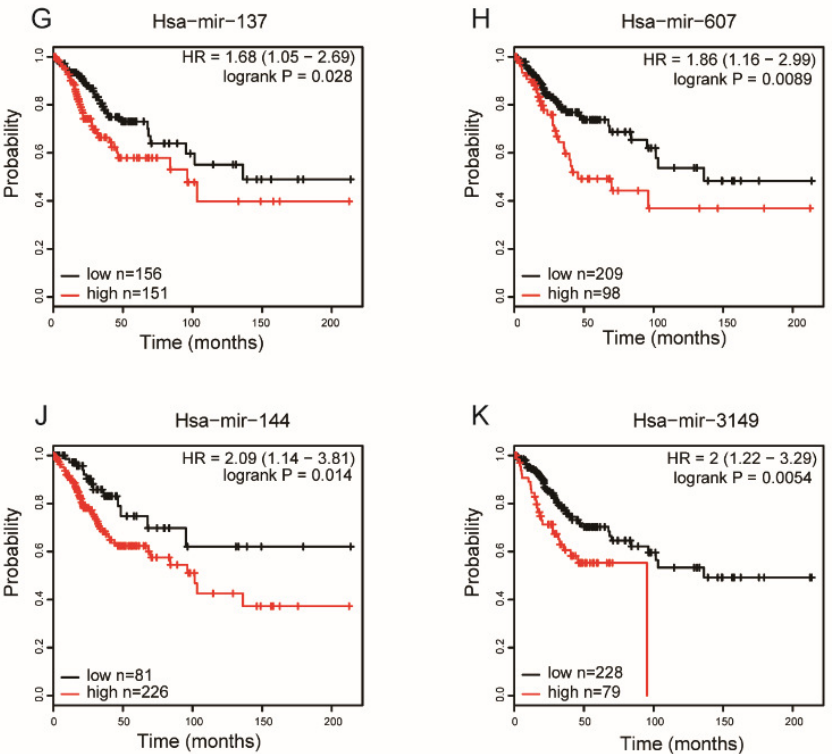

$\mathrm{K}$

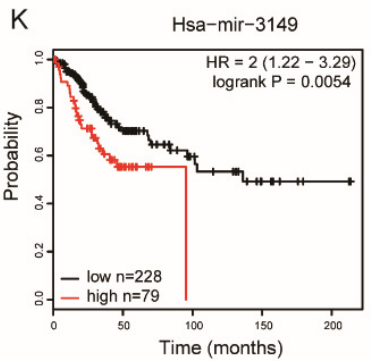

B

D

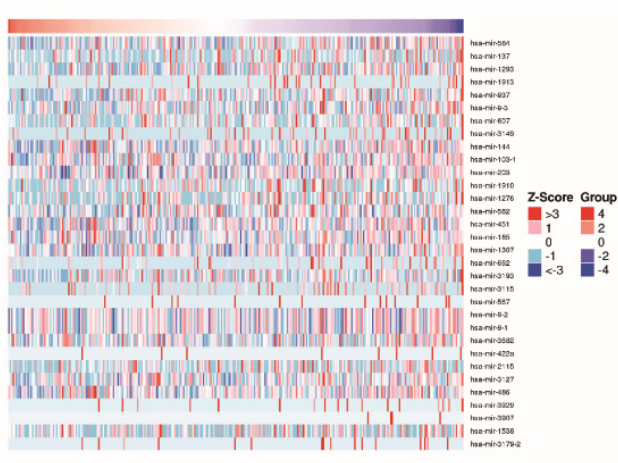

$\mathrm{F}$

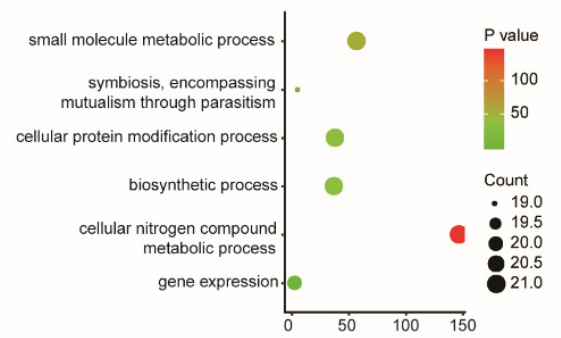

Cellcular Components

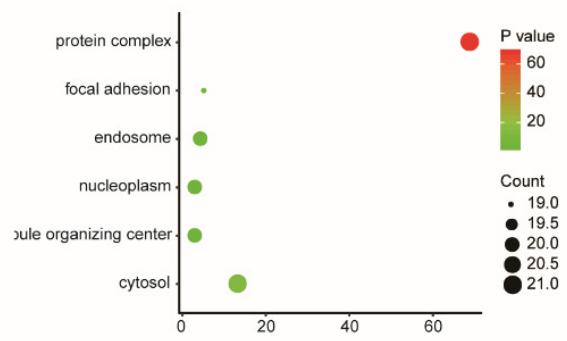

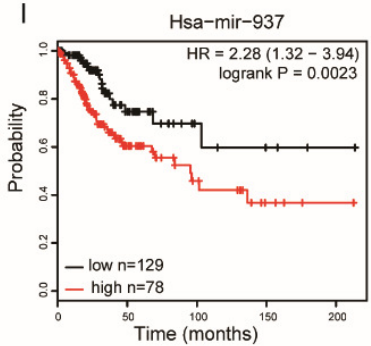

L

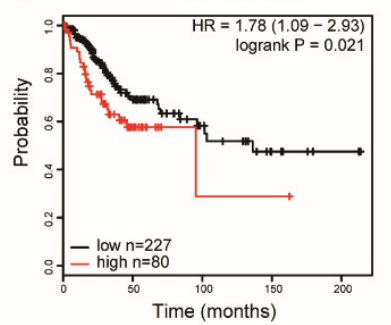


541 (A) Pearson test was used to analyze correlations between OTUD5, and differentially expressed 542 miRNAs d in CESC. (B) Heat maps showing genes negatively correlated with OTUD5 in CESC 543 (TOP 33). (C-F) Enrichment analysis of miRNAs negatively correlated with OTUD5 in cervical 544 cancer. The groups display the enrichment results of the top 9 miRNAs that were altered in CESC. 545 (C) Pathway analysis. (D) Biological processes. (E) Molecular functions. (F) Cellular components. 546 (G-L) Impact of miRNA expression on overall survival in CESC patients (Kaplan-Meier Plotter): 547 hsa-mir-137, hsa-mir-1913, hsa-mir-937, hsa-mir-607, hsa-mir-3149 and hsa-mir-144. 


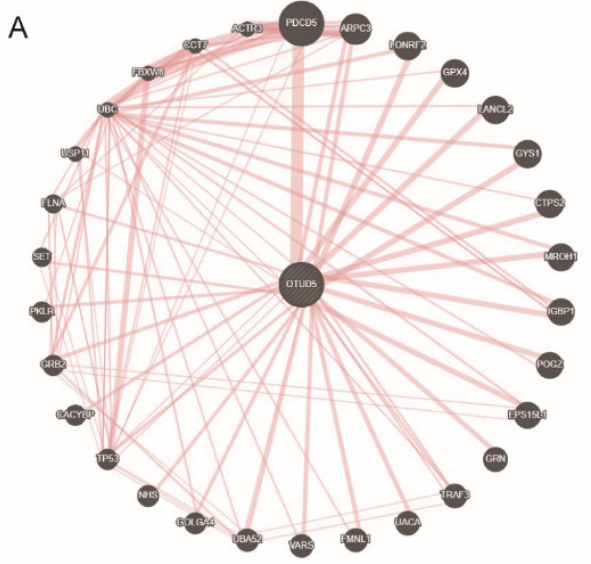

C

Biogolical Processes

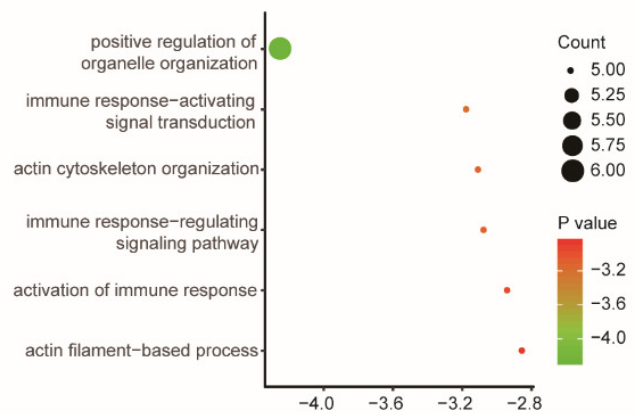

B

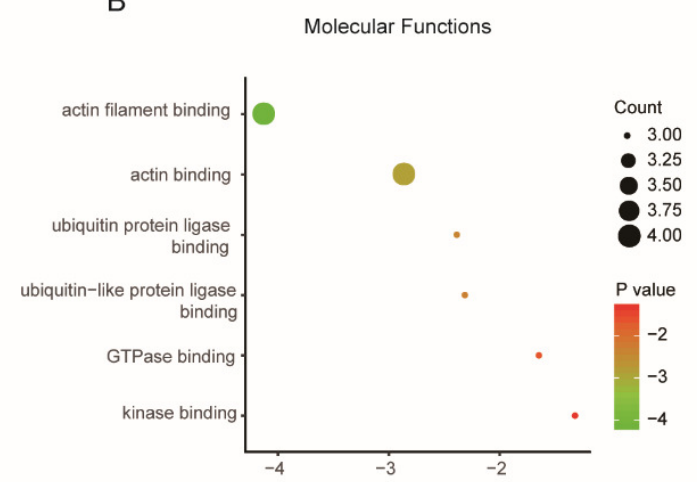

D

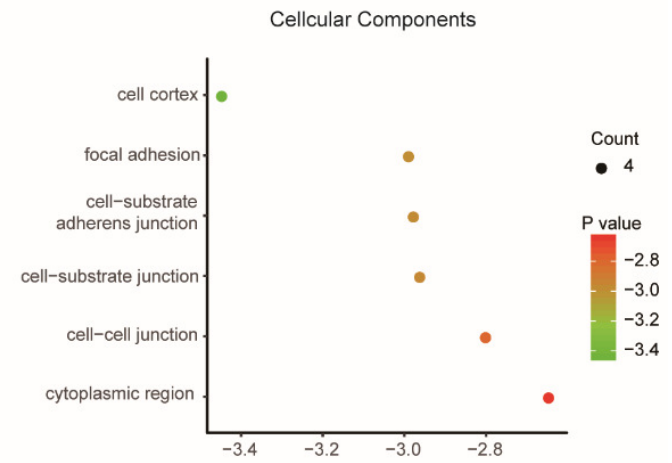

561

FIG6 . Functional annotations of OTUD5 interacting proteins.

(A)Protein-protein interaction network of OTUD5 (GeneMANIA). (B-D) Enrichment analysis of the proteins interacting with OTUD5. The groups display the enrichment results of the top 30 genes interacting with OTUD5 in CESC. (B) Molecular functions (C) Biological processes. (D) Cellular components. 
A

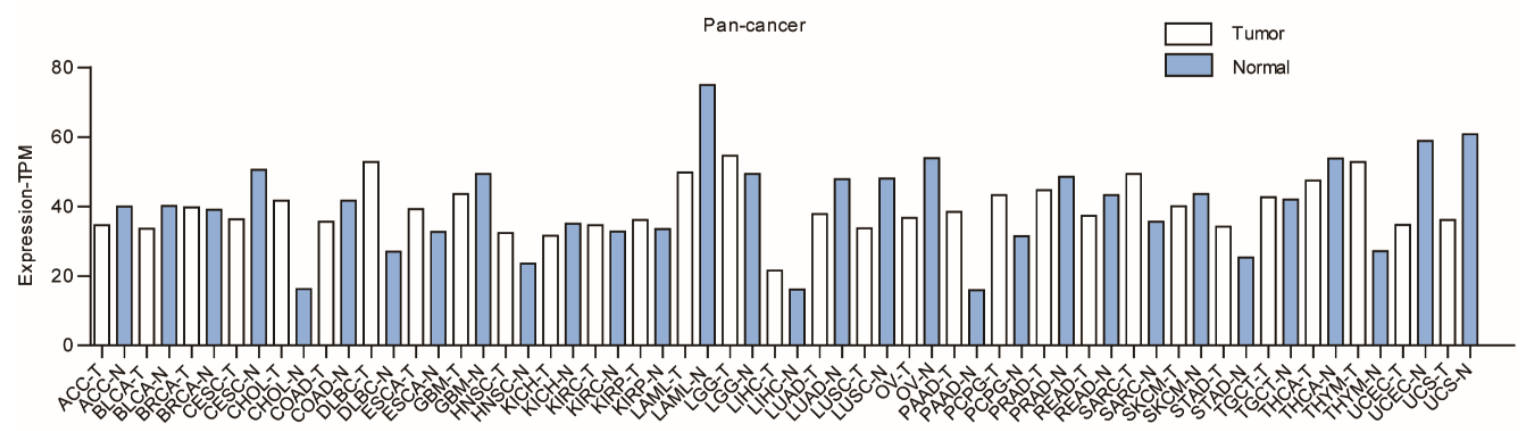

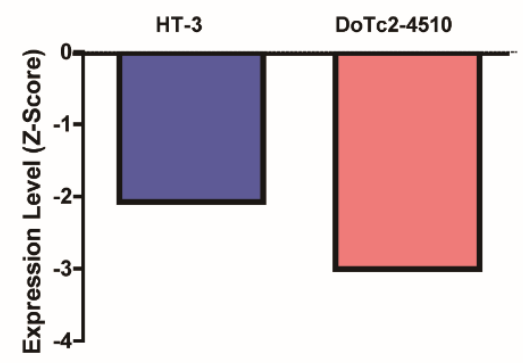

571

572

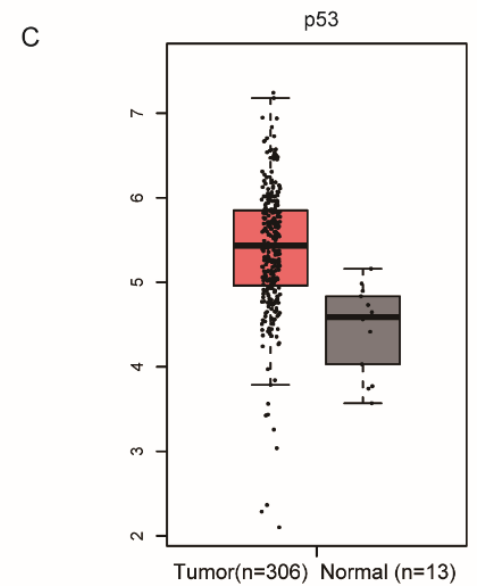

Supplementary FIG. 1 The expression of OTUD5 and p53.

(A) Expression of OTUD5 in 31 types of normal and tumor samples. Transcripts per million (TPM) of OTUD5 in CESC (TCGA) from the GEPIA database.

(B) Expression of OTUD5 in cervical cancer cell lines (HT-3 and DoTc2-4510) through COSMIC database analysis.

(C) Box plot showing the transcripts per million (TPM) of p53 in CESC (TCGA) from the GEPIA database. 
582 TABLE 1 Co-expression with OTUD5 in CESC from LinkedOmics.

\begin{tabular}{|c|c|c|c|c|}
\hline \multirow[t]{2}{*}{ Genes } & \multicolumn{2}{|c|}{$\begin{array}{c}\text { Gene expression } \\
\log 2(\mathrm{TPM}+1) \text { for log-scale. }\end{array}$} & \multirow[t]{2}{*}{ Prognose } & \multirow[t]{2}{*}{ Correlation } \\
\hline & Normal & Tumor & & \\
\hline GPKOW & 4.2 & 4.5 & Favorable & 0.79 \\
\hline CCDC22 & 3.8 & 2.4 & Favorable & 0.76 \\
\hline WDR45" & 6.3 & 5.6 & Favorable & 0.65 \\
\hline WDR13" & 7.1 & 6.5 & Favorable & 0.38 \\
\hline ARAF $\#$ & 5.7 & 5.6 & Favorable & 0.48 \\
\hline GRIPAP1" & 5.3 & 5.0 & Favorable & 0.84 \\
\hline UXT & 7.1 & 7.3 & Favorable & 0.54 \\
\hline RBM10 $\#$ & 5.2 & 5.2 & Favorable & 0.73 \\
\hline TSR2 & 5.5 & 5,5 & Unfavorable & 0.73 \\
\hline TIMM17B & 5.4 & 6.2 & Favorable & 0.57 \\
\hline PQBP1 $\#$ & 6.5 & 6.4 & Favorable & 0.51 \\
\hline NDUFB11 & 6.0 & 7.1 & Favorable & 0.40 \\
\hline HSD17B10 & 6.2 & 7.0 & Favorable & 0.51 \\
\hline TBC1D25" & 3.4 & 3.1 & Favorable & 0.70 \\
\hline SUV39H1 & 2.2 & 3.7 & Favorable & 0.67 \\
\hline FTSJ1 & 4.9 & 5.7 & Favorable & 0.50 \\
\hline PHKA2 ${ }^{\#}$ & 4.7 & 3.8 & Favorable & 0.65 \\
\hline SPIN2B\# & 3.7 & 2.9 & $\mathrm{~N} / \mathrm{A}$ & 0.51 \\
\hline USP11 ${ }^{\#}$ & 6.4 & 5.0 & Favorable & 0.72 \\
\hline IGBP1 ${ }^{\#}$ & 6.0 & 5.4 & $\mathrm{~N} / \mathrm{A}$ & 0.50 \\
\hline APEX2 & 3.3 & 4.5 & Favorable & 0.58 \\
\hline LAS1L & 5.1 & 5.0 & Favorable & 0.51 \\
\hline RNF113A & 3.6 & 4.0 & Favorable & 0.45 \\
\hline FAM104B & 4.1 & 4.3 & Favorable & 0.45 \\
\hline TFE3 & 5.6 & 4.6 & Favorable & 0.70 \\
\hline TSPYL2 & 7.5 & 3.7 & Unfavorable & 0.63 \\
\hline ELK1 & 4.0 & 4.5 & N/A & 0.71 \\
\hline PRAF2 & 5.5 & 4.5 & Favorable & 0.48 \\
\hline IDH3G & 5.9 & 5.9 & Favorable & 0.41 \\
\hline MAGED2\# & 7.5 & 6.2 & $\mathrm{~N} / \mathrm{A}$ & 0.60 \\
\hline HDAC6 ${ }^{\#}$ & 6.4 & 5.1 & Favorable & 0.58 \\
\hline PDHA1 & 6.6 & 7.0 & Favorable & 0.49 \\
\hline CDK16 & 5.7 & 6.4 & Favorable & 0.47 \\
\hline LOC550643 & 4.8 & 5.0 & N/A & \\
\hline SLC25A14 $\#$ & 3.8 & 3.6 & Favorable & 0.55 \\
\hline PIN4 & 4.2 & 4.7 & $\mathrm{~N} / \mathrm{A}$ & 0.39 \\
\hline
\end{tabular}




\begin{tabular}{lllll} 
EBP & 4.6 & 7.3 & Favorable & 0.36 \\
LOC401588 & 2.5 & 1.4 & Favorable & 0.59 \\
USP27X $^{\#}$ & 2.0 & 1.6 & Favorable & 0.66 \\
SPSB3 $^{\#}$ & 6.2 & 5.4 & Favorable & 0.42 \\
CXorf40A & 4.0 & 4.2 & Favorable & 0.41 \\
C17orf59 & 2.8 & 2.6 & Favorable & 0.35 \\
SLC35A2 & 4.0 & 4.8 & N/A & 0.63 \\
FAM156A & 4.6 & 3.3 & Favorable & 0.53 \\
GABARAP & 9.7 & 9.0 & Favorable & 0.39 \\
NKAP & 2.8 & 3.0 & Favorable & 0.49 \\
RBMX2 $^{\#}$ & 4.7 & 4.5 & Favorable & 0.43 \\
UBA1 $^{\text {MSL3 }}{ }^{\#}$ & 7.1 & 7.3 & N/A & 0.60 \\
\hline
\end{tabular}

TABLE 2 Overall survival of miRNA that target OTUD5 in CESC.

\begin{tabular}{|c|c|c|c|c|c|}
\hline miRNA & $\begin{array}{l}\text { Low } \\
\text { expression }\end{array}$ & $\begin{array}{l}\text { High } \\
\text { expression }\end{array}$ & Prognose & $\begin{array}{l}P \\
\text { value }\end{array}$ & HR \\
\hline Has-mir-584 & $\mathrm{n}=22$ & $\mathrm{n}=185$ & $\mathrm{~N} / \mathrm{A}$ & $\begin{array}{l}0.190 \\
0\end{array}$ & $\begin{array}{l}1.39(0.85- \\
2.29)\end{array}$ \\
\hline Has-mir-137 & $n=156$ & $n=151$ & $\begin{array}{l}\text { Unfavorabl } \\
\mathrm{e}\end{array}$ & $\begin{array}{l}0.028 \\
0\end{array}$ & $\begin{array}{l}1.68(1.05- \\
2.69)\end{array}$ \\
\hline Has-mir-1293 & $\mathrm{n}=83$ & $n=224$ & $\mathrm{~N} / \mathrm{A}$ & $\begin{array}{l}0.180 \\
0\end{array}$ & $\begin{array}{l}0.71(0.43- \\
1.18\end{array}$ \\
\hline Has-mir-1913 & $n=227$ & $\mathrm{n}=80$ & $\begin{array}{l}\text { Unfavorabl } \\
\mathrm{e}\end{array}$ & $\begin{array}{l}0.021 \\
0\end{array}$ & $\begin{array}{l}1.78(1.09- \\
2.93)\end{array}$ \\
\hline Has-mir-937 & $n=129$ & $\mathrm{n}=178$ & $\begin{array}{l}\text { Unfavorabl } \\
\mathrm{e}\end{array}$ & $\begin{array}{l}0.002 \\
3\end{array}$ & $\begin{array}{l}2.28(1.32- \\
3.94)\end{array}$ \\
\hline Has-mir-9-3 & $\mathrm{n}=76$ & $n=231$ & Favorable & $\begin{array}{l}0.031 \\
0\end{array}$ & $\begin{array}{l}0.58(0.35- \\
0.96)\end{array}$ \\
\hline Has-mir-607 & $n=209$ & $\mathrm{n}=98$ & $\begin{array}{l}\text { Unfavorabl } \\
\mathrm{e}\end{array}$ & $\begin{array}{l}0.008 \\
9\end{array}$ & $\begin{array}{l}1.86(1.16- \\
2.99)\end{array}$ \\
\hline Has-mir-3149 & $n=228$ & $n=79$ & $\begin{array}{l}\text { Unfavorabl } \\
\mathrm{e}\end{array}$ & $\begin{array}{l}0.005 \\
4\end{array}$ & $\begin{array}{l}2.00(1.22- \\
3.29)\end{array}$ \\
\hline Has-mir-144 & $\mathrm{n}=81$ & $n=226$ & $\begin{array}{l}\text { Unfavorabl } \\
\mathrm{e}\end{array}$ & $\begin{array}{l}0.014 \\
0\end{array}$ & $\begin{array}{l}2.09(1.14- \\
3.81)\end{array}$ \\
\hline $\begin{array}{l}\text { Has-mir-103- } \\
1\end{array}$ & $n=159$ & $\mathrm{n}=148$ & $\mathrm{~N} / \mathrm{A}$ & $\begin{array}{l}0.042 \\
0\end{array}$ & $\begin{array}{l}1.62(1.01- \\
2.61)\end{array}$ \\
\hline
\end{tabular}


587 TABLE 3 Transcription factor of OTUD5 in QIAGEN, ConTra v3 and PROMO.

\begin{tabular}{|c|c|c|c|c|c|c|c|}
\hline Database & Gene & Correlation & P value & Database & Gene & Correlation & P value \\
\hline \multirow[t]{4}{*}{ QIAGEN } & EST1 & 0.12 & 0.038 & PROMO & IRF-2\# & 0.33 & $2.7 e-09$ \\
\hline & NKX2-5 & -0.07 & 0.250 & & RXRA & 0.10 & 0.084 \\
\hline & PAX4 & -0.02 & 0.680 & & YY1\# & 0.21 & 0.001 \\
\hline & PPARG & 0.07 & 0.250 & & XBP1 & 0.13 & 0.021 \\
\hline \multirow[t]{15}{*}{ ConTra v3 } & SP1\# & 0.34 & $6.2 e-10$ & & Cebpb & -0.04 & 0.480 \\
\hline & $\mathrm{SP} 2^{\#}$ & 0.40 & $7.1 \mathrm{e}-13$ & & PAX5 & 0.02 & 0.720 \\
\hline & SP3\# & 0.26 & $6.1 e-06$ & & TFIID\# & 0.21 & 0.001 \\
\hline & $\mathrm{SP} 4^{\#}$ & 0.32 & $7.6 e-09$ & & FOXP3\# & 0.27 & $1.9 e-06$ \\
\hline & MAZ\# & 0.23 & $4.0 e-05$ & & GR & 0.12 & 0.039 \\
\hline & MAZR \# & 0.37 & $1.9 e-11$ & & GTF2I $\#$ & 0.31 & $4.7 e-08$ \\
\hline & KLF4 & 0.02 & 0.760 & & $N F-1^{\#}$ & 0.32 & $9.1 \mathrm{e}-09$ \\
\hline & KLF5 & 0.06 & 0.300 & & ESR1 & 0.13 & 0.019 \\
\hline & KLF16 & -0.02 & 0.720 & & SLEB11 & -0.02 & 0.780 \\
\hline & TCN1 & -0.11 & 0.058 & & ETS1 & 0.12 & 0.038 \\
\hline & ELF4\# & 0.28 & $4.2 \mathrm{e}-07$ & & ELK1\# & 0.71 & $2.2 \mathrm{e}-47$ \\
\hline & & & & & TFAP2A & 0.10 & 0.100 \\
\hline & & & & & SP1\# & 0.34 & 0.038 \\
\hline & & & & & HMGA1 & 0.10 & 0.130 \\
\hline & & & & & P53\# & 0.37 & $2.6 e-11$ \\
\hline
\end{tabular}

588

589

590

591

TABLE 4 Kinase of OTUD5 in CESC from LinkedOmics.

\begin{tabular}{lllll}
\hline Gene & size & FDR & \multicolumn{2}{l}{$\begin{array}{l}\text { Gene expression log2(TPM + 1) } \\
\text { for log-scale. }\end{array}$} \\
\hline & & & Normal & Tumor \\
PLK1 & 38 & 0.0075271 & 1.6 & 5.3 \\
ATM & 50 & 0.015054 & 4.3 & 2.7 \\
PIK3CA & 4 & 0.067242 & 3.2 & 3.2 \\
CDK5 & 28 & 0.074669 & 3 & 4.2 \\
CHEK1 & 46 & 0.078282 & 1.4 & 4.2 \\
\hline
\end{tabular}

592

593

594 
595

596

TABLE 5 Ubiquitin ligase of OTUD5 in CESC from UbiBrowser.

\begin{tabular}{|c|c|c|c|c|c|c|c|}
\hline \multirow[t]{2}{*}{ Genes } & \multirow[t]{2}{*}{ SCORE } & \multicolumn{2}{|c|}{$\begin{array}{c}\text { Gene expression } \\
\log 2(\mathrm{TPM}+1) \text { for } \\
\text { log-scale. }\end{array}$} & \multirow[t]{2}{*}{ Genes } & \multirow[t]{2}{*}{ SCORE } & \multicolumn{2}{|c|}{$\begin{array}{c}\text { Gene expression } \\
\log 2(\mathrm{TPM}+1) \\
\text { for log-scale. }\end{array}$} \\
\hline & & Normal & Tumor & & & Normal & Tumor \\
\hline RNF180 & 0.719 & 1.9 & 0.1 & FBXO3 & 0.623 & 4.6 & 4.0 \\
\hline NEDD4L ${ }^{\#}$ & 0.705 & 3.0 & 4.7 & HERC2 & 0.623 & 5.1 & 4.8 \\
\hline RNF6 $^{\#}$ & 0.694 & 3.4 & 3.9 & TRIM11 & 0.610 & 3.8 & 4.6 \\
\hline MARCH1 & 0.694 & 1.8 & 1.3 & KLHL13 & 0.610 & 4.1 & 1.9 \\
\hline MDM2 & 0.668 & 5.0 & 6.0 & MARCH8 & 0.610 & 3.6 & 2.8 \\
\hline SYVN1 & 0.667 & 5.3 & 4.8 & RFPL4A & 0.610 & - & - \\
\hline PIAS4 & 0.648 & 3.7 & 3.8 & MARCH7 & 0.608 & 5.2 & 5.3 \\
\hline MIB1 & 0.642 & 3.4 & 2.8 & RNF216 & 0.608 & 4.1 & 3.4 \\
\hline ZC3HC1 & 0.623 & 3.7 & 3.6 & MIB2 & 0.594 & 6.1 & 5.1 \\
\hline & & & & UBE3C & 0.594 & 4.6 & 4.6 \\
\hline
\end{tabular}

597

598

599 
Figure 1

The expression of OTUD5 in CESC.

(A) Overall survival of CESC patients (TCGA). (B) Box plot showing the transcripts per million (TPM) of OTUD5 in CESC (TCGA) from the GEPIA database. (C-D) Scatter plot showing mutation and copy number alteration of OTUD5 in CESC patients using CBioPortal. (E) The correlation of OTUD5 and methylation in CESC data from MethHC (http://methhc.mbc.nctu.edu.tw/php/index.php). (F) Box plot showing OTUD5 methylation in CESC via UALCAN. The beta value indicates the level of DNA methylation ranging from 0 (unmethylated) to 1 (fully methylated).

A

OTUD5 (Overall Surivial)
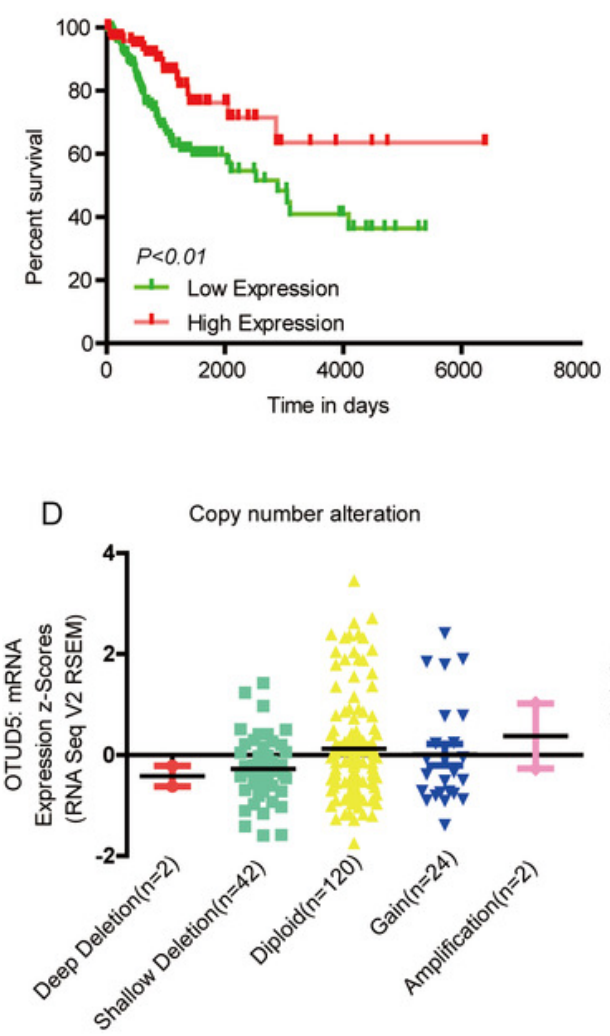

B
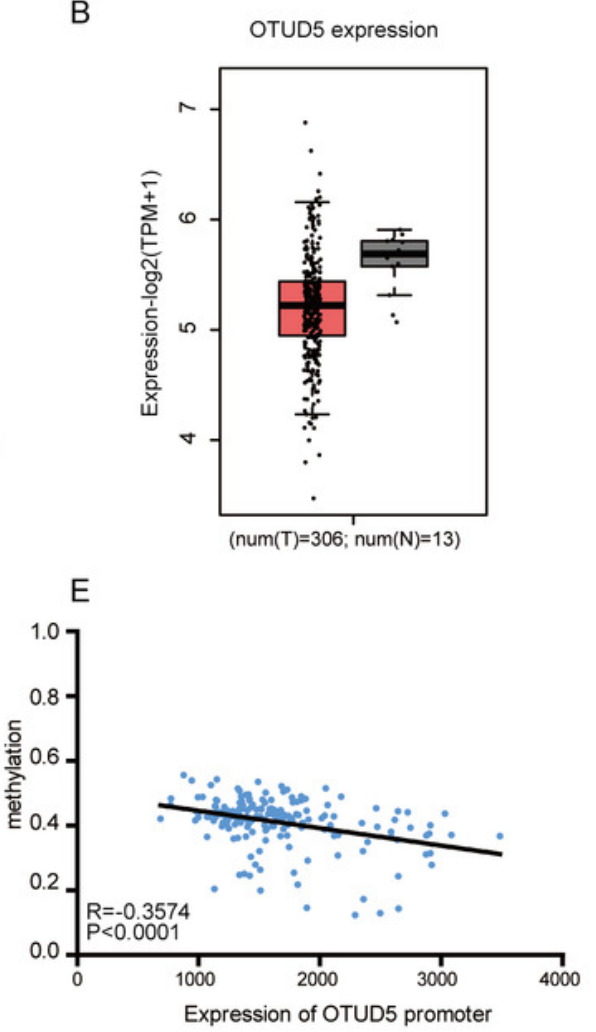

C

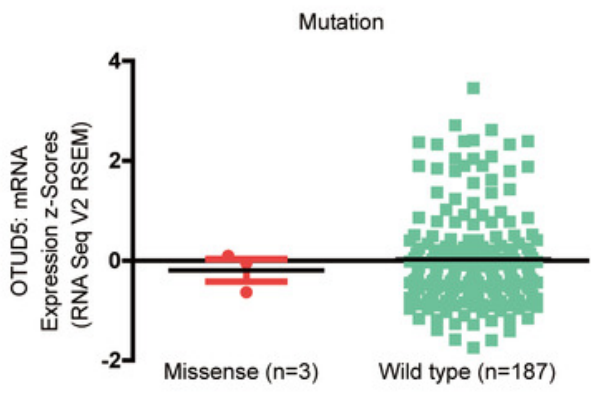

$\mathrm{F}$ Promoter methylation level of OTUD5 in CESC

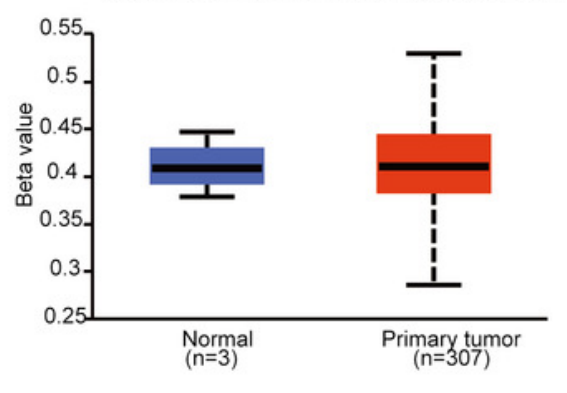




\section{Figure 2}

The relationship between OTUD5 expression and clinical pathology of cervical cancer.

(A) Boxplot showing relative expression of OTUD5 in tumor stage (stage1, stage2, stage3, stage4 or stage5) from CESC patients via UALCAN. (B) Boxplot showing relative expression of OTUD5 in nodal metastasis status (Normal, N0 or N1) from normal and CESC patients via UALCAN. (C-E)Boxplot showing relative expression of OTUD5 in age (aged 21-40, 41-60, 61-80, or 81-100 years), weight (Normal, Normal weight, Extreme weight, Obese or Extreme obese) and race (Normal, Caucasian, African-American or Asian) from CESC patients via UALCAN. (F) Box plot showing OTUD5 copy number in The Cancer Genome Atlas (TCGA) Scotto Cervix via ONCOMINE. (G) Box plot showing Epithelial-Mesenchymal Transition (EMT) in CESC patients affected by OTUD5 in GEPIA database. (H) Box plot showing Hormone in CESC patients affected by OTUD5 in GEPIA database. (I) Box plot showing PI3K-AKT pathway in CESC patients affected by OTUD5 in GEPIA database. 
A

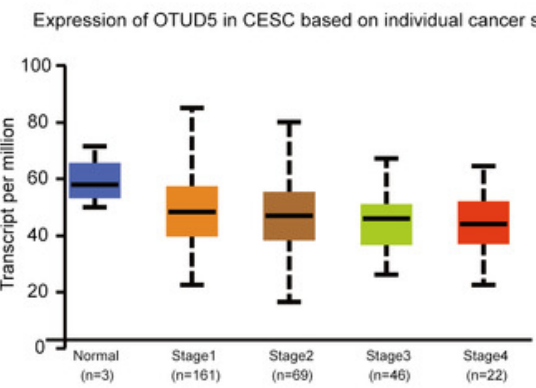

D

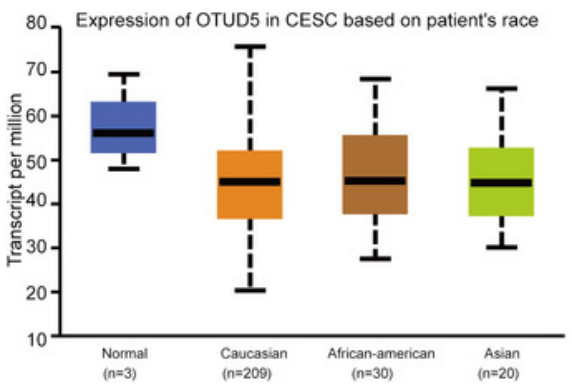

G

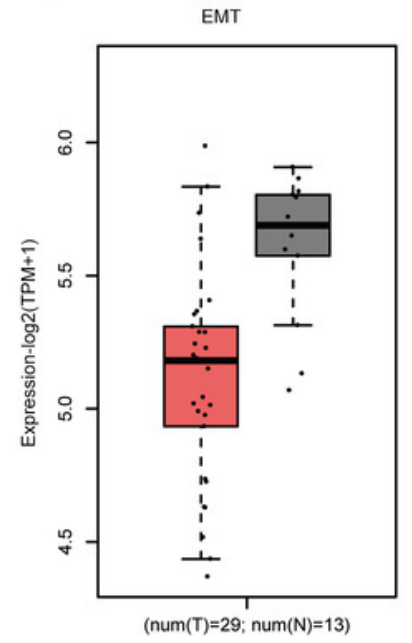

B

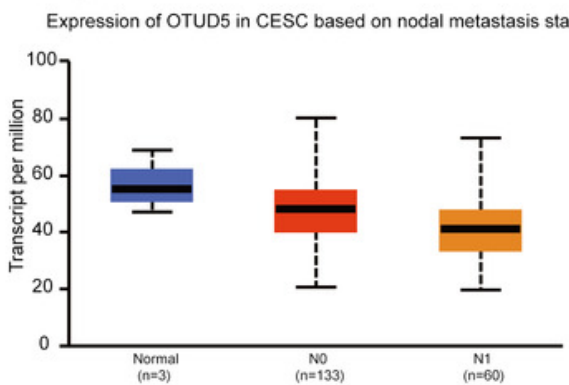

E

Expression of OTUD5 in CESC based on patient's weight
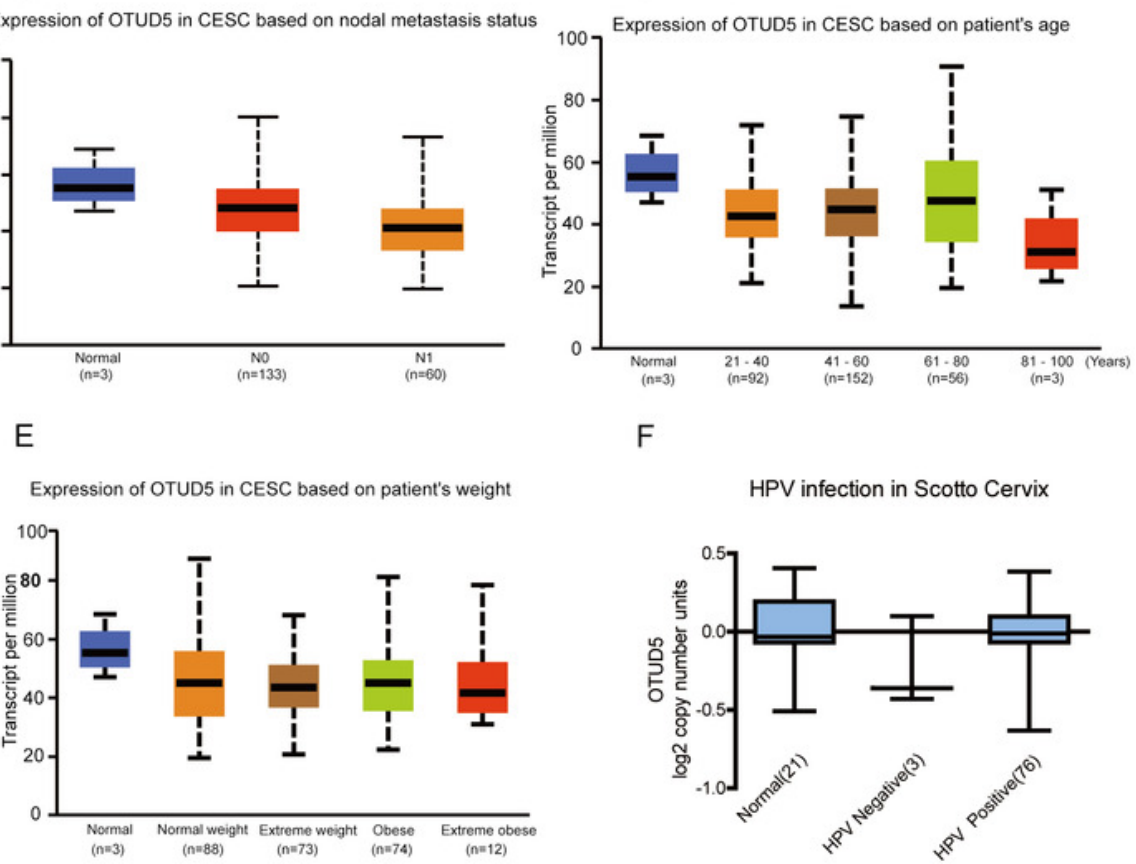

$\mathrm{F}$

HPV infection in Scotto Cervix

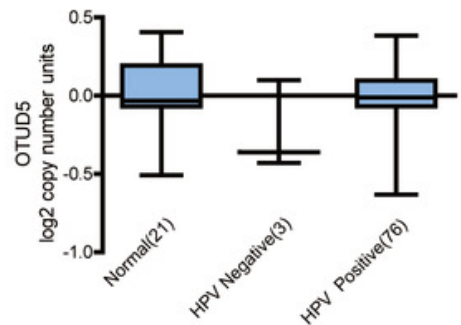

$\mathrm{H}$

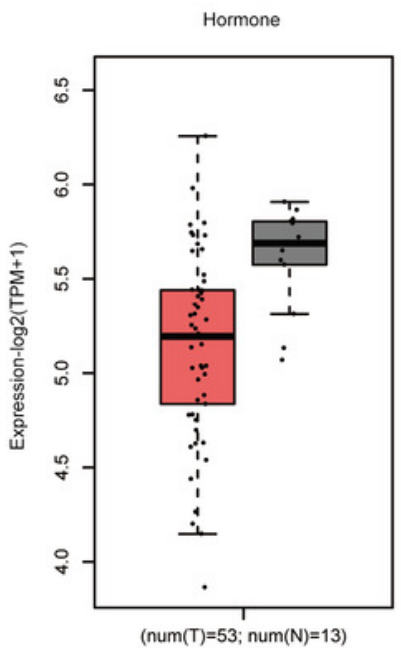

I

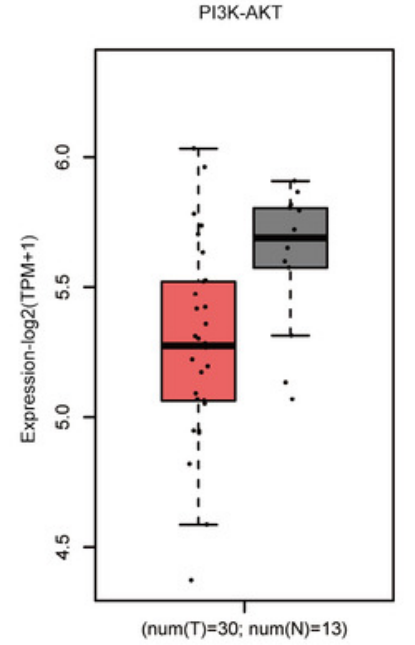


Figure 3

GO Functional Annotation and Pathway Enrichment of OTUD5 Co-Expressed Genes in TCGA-CESC.

(A) A Pearson test was used to analyze correlations between OTUD5, and genes differentially expressed in CESC. (B) Heat maps showing genes positively correlated with OTUD5 in CESC (TOP 50). (C-D) Enrichment analysis of the genes altered in the OTUD5 co-expression genes in cervical cancer. The box plot displays the enrichment results of the top 22 genes altered in the OTUD5 co-expression genes in CESC. (C) Biological processes. (D) Molecular functions.

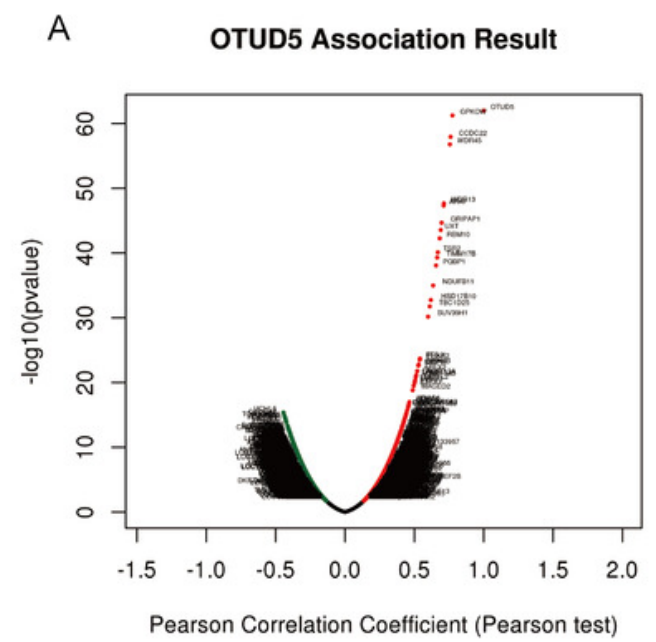

C

Biogoical Processes

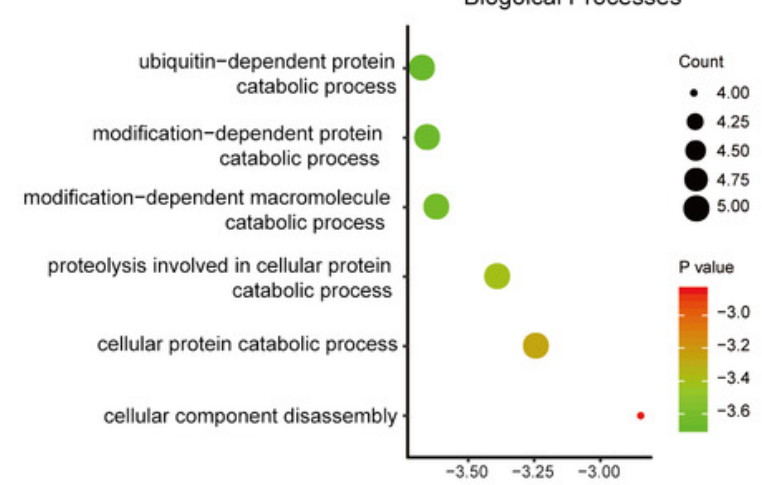

B

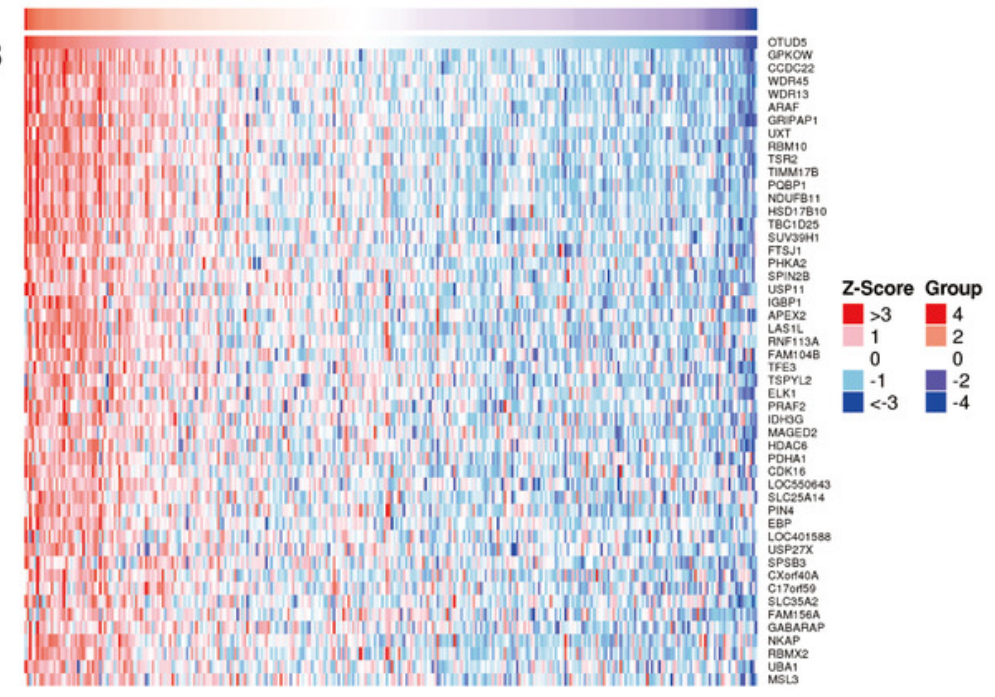

D

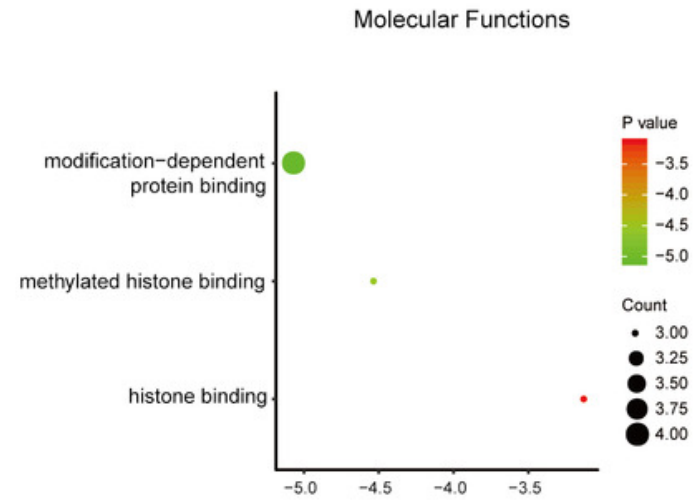


Figure 4

USP11, RBM10, WDR45 and GRIPAP1 have associated with OTUD5.

(A-D) The scatter plot shows Pearson correlation of OTUD5 expression with expression USP11, RBM10, WDR45 and GRIPAP1 in CESC patients (linkedomics). (E-H) Overall survival curve showing USP11, RBM10, WDR45 and GRIPAP1 in CESC patients (linkedomics), low expression ( $n=137)$, high expression $(n=136)$. (I-L) mRNA of USP11, RBM10, WDR45 and GRIPAP1 were detected by qRT-PCR when knocked down OTUD5 in HeLa cells using siRNA.
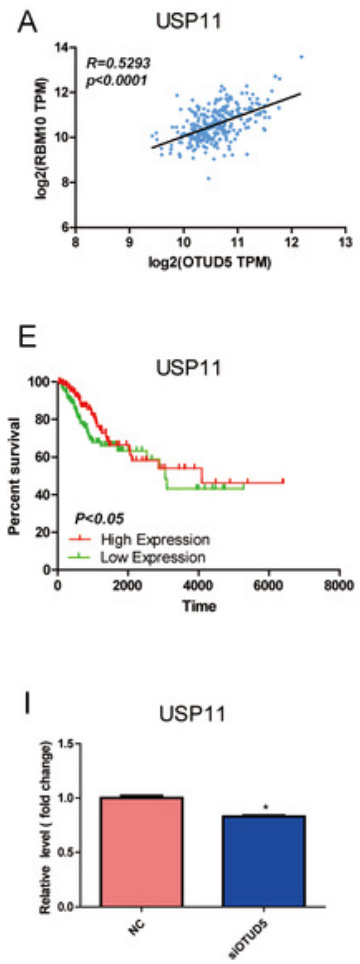
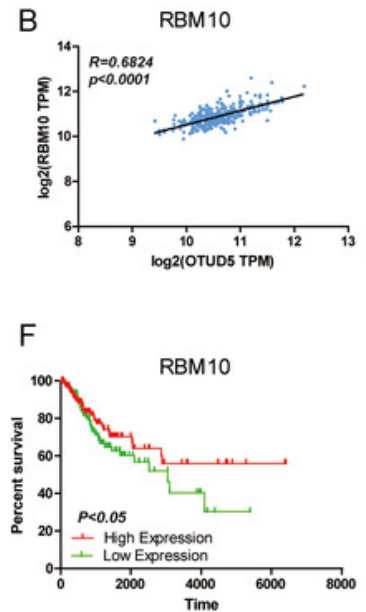

$\mathrm{J}$

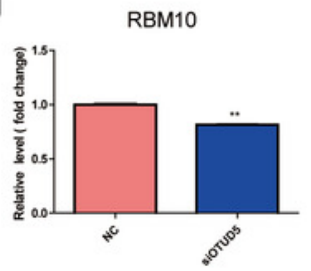

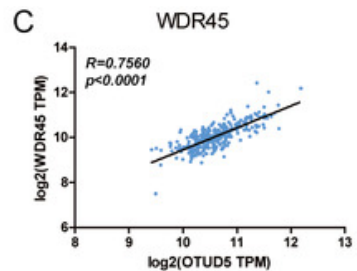
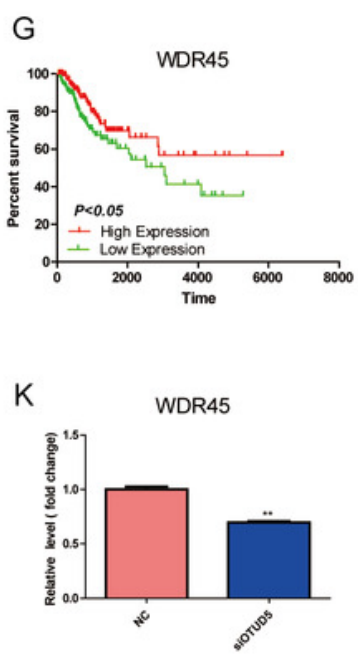
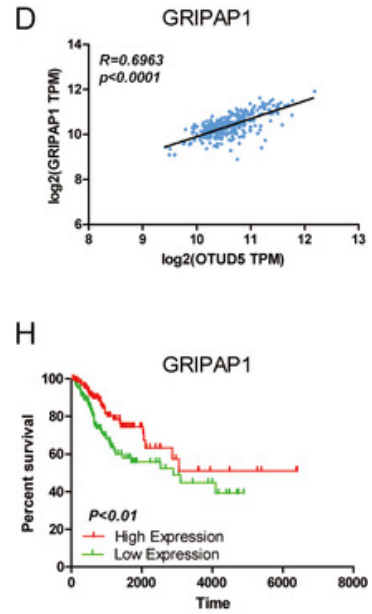

$\mathrm{L}$

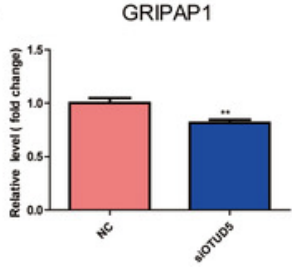




\section{Figure 5}

miRNA-OTUD5 network in CESC.

(A) A Pearson test was used to analyze correlations between OTUD5, and miRNA differentially expressed in CESC. (B) Heat maps showing genes negatively correlated with OTUD5 in CESC (TOP 33). (C-F) Enrichment analysis of the miRNA negatively correlated with OTUD5 in cervical cancer. The groups display the enrichment results of the top 9 miRNA that was altered in CESC. (C) pathway. (D) biological processes. (E) molecular functions. (F) cellular components. (G-L) Impact of miRNA expression on overall survival in CESC patients (KaplanMeier Plotter): hsa-mir-137, hsa-mir-1913, hsa-mir-937, hsa-mir-607, hsa-mir-3149 and hsamir-144. 
A

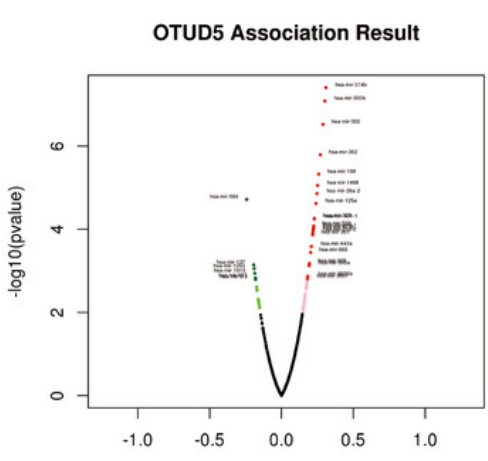

C

Pearson Correlation Coefficient (Pearson test)

Pathway
B

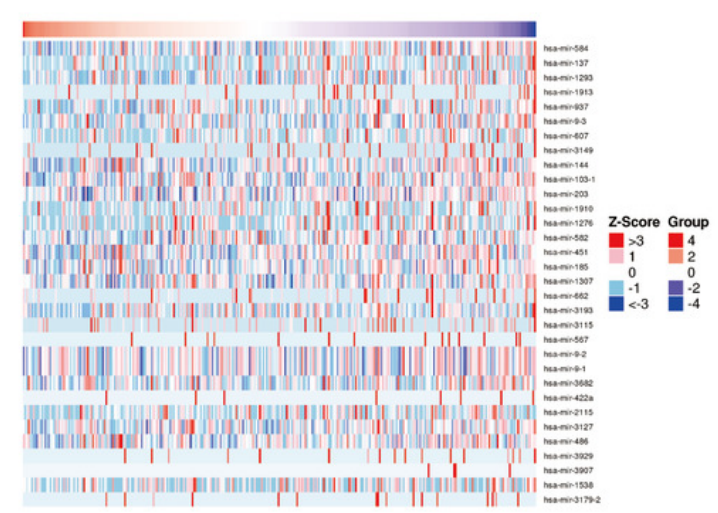

D

Biogolical Processes

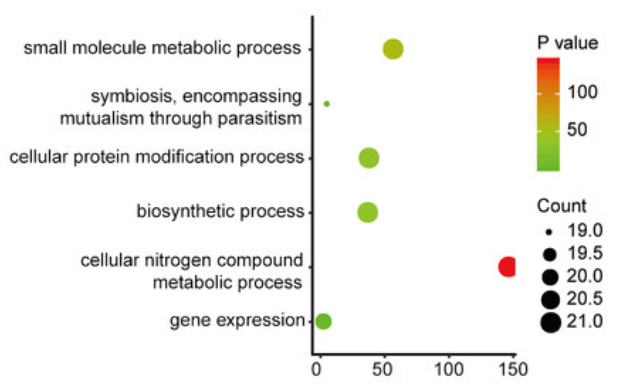

Cellcular Components

$\mathrm{F}$
$\mathrm{F}$
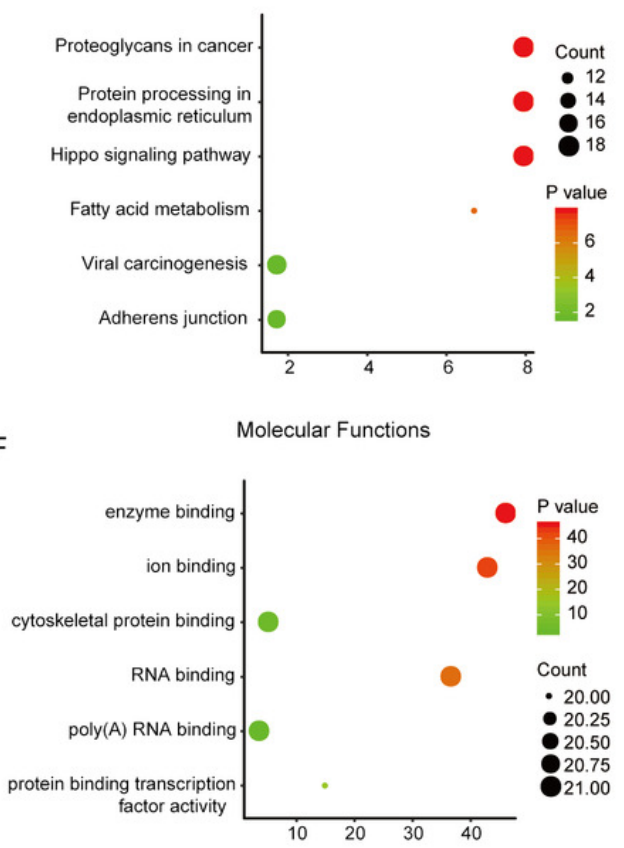

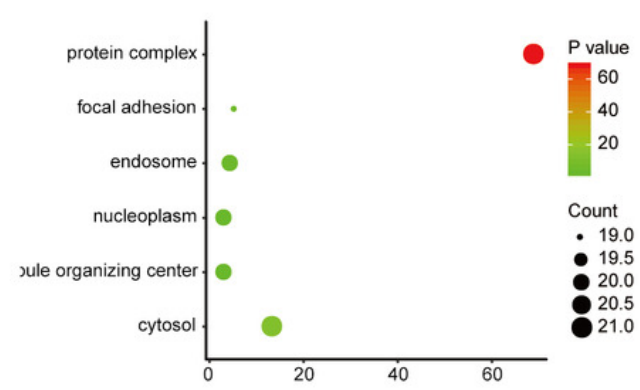

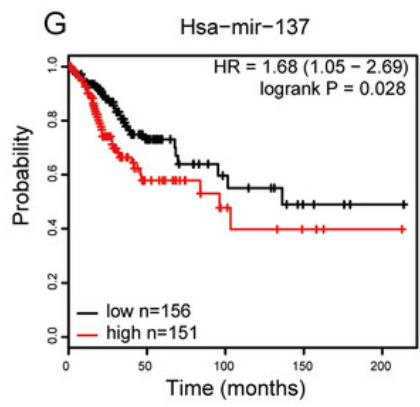

$\mathrm{H}$
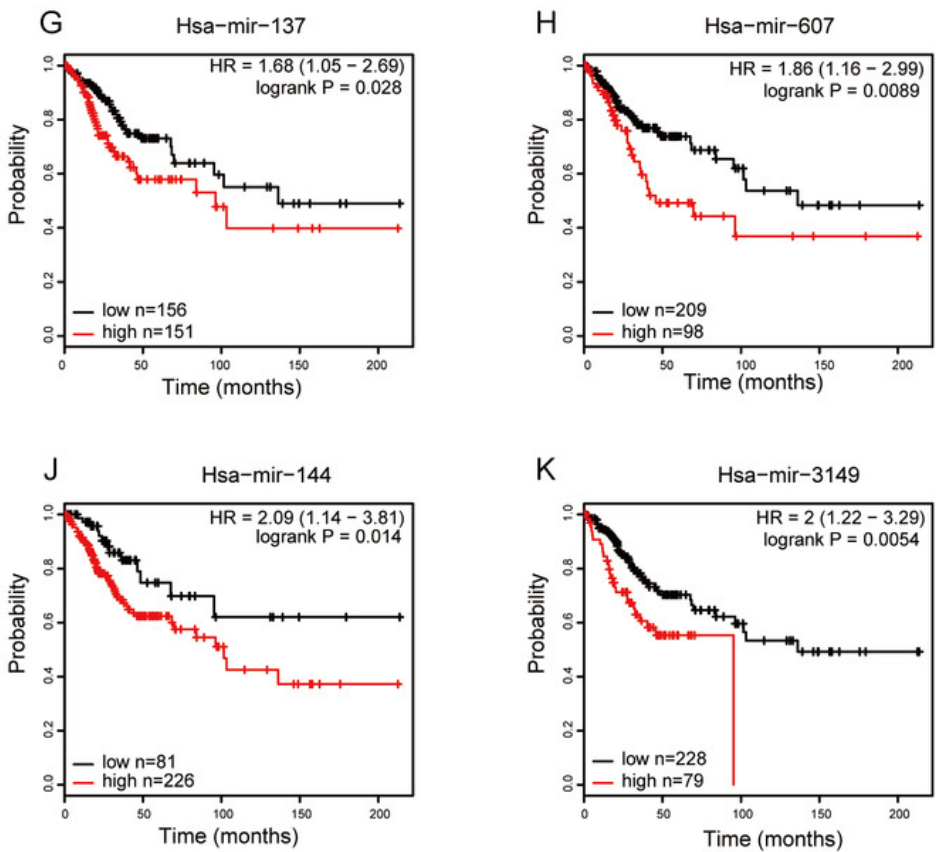

$\mathrm{K}$

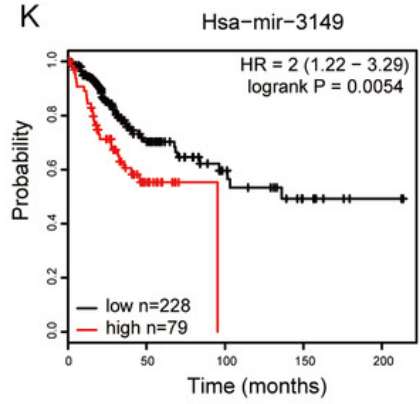

I Hsa-mir-937

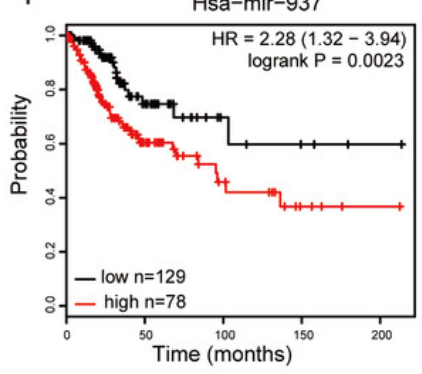

L

Hsa-mir-1913

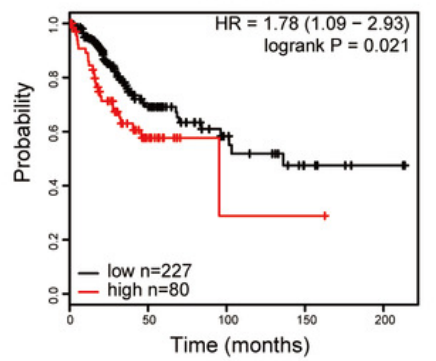


Figure 6

Functional annotations of OTUD5 interaction proteins.

(A) Protein-protein interaction network of OTUD5 (GeneMANIA). (B-D) Enrichment analysis of the protein interacted with OTUD5. The groups display the enrichment results of the top 30 interaction genes with OTUD5 in CESC. (B) Molecular functions (C) Biological processes. (D) Cellular components.

A

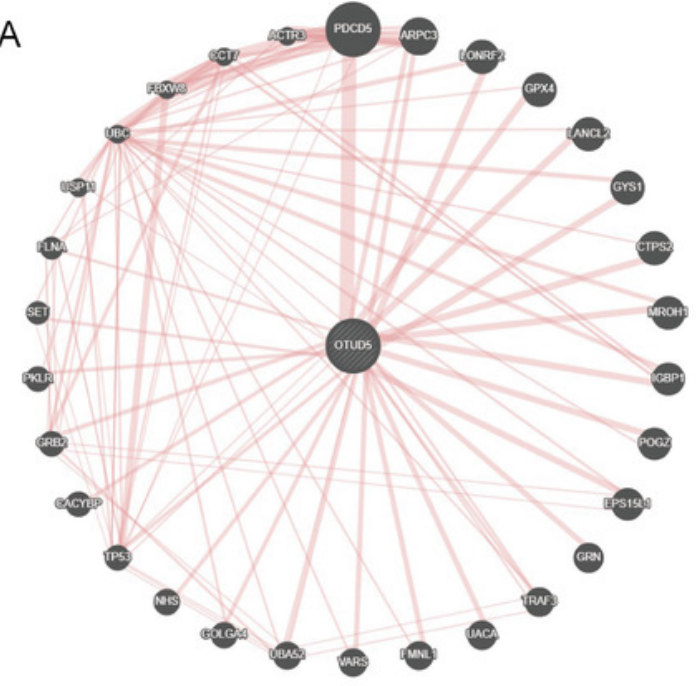

C

Biogolical Processes

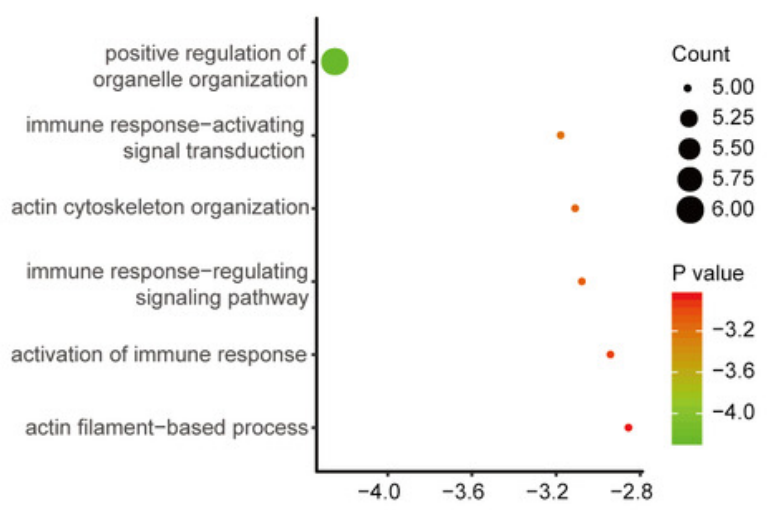

$\mathrm{B}$

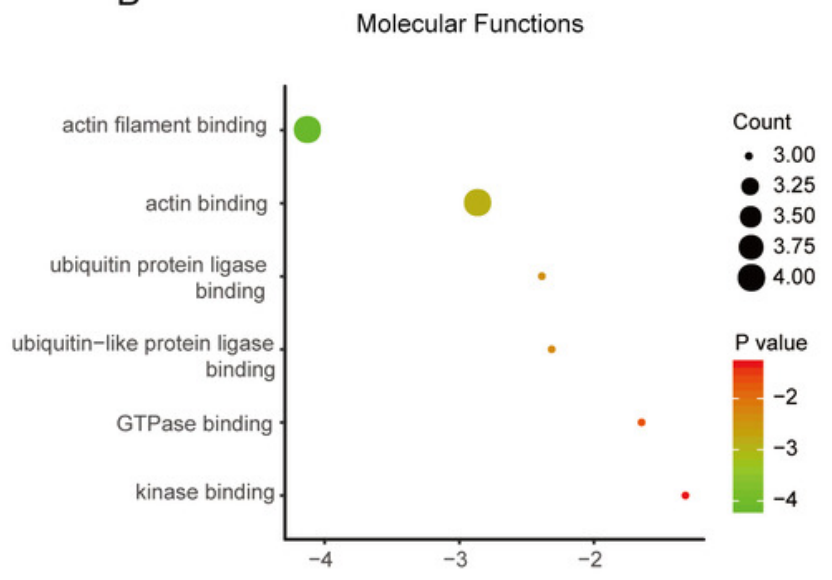

D

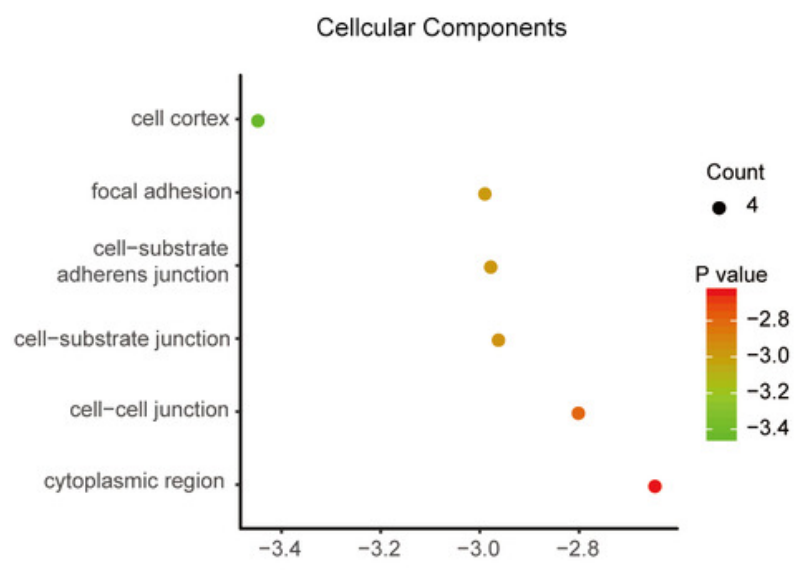


Table $\mathbf{1}$ (on next page)

Co-expression with OTUD5 in CESC from LinkedOmics. 


\begin{tabular}{|c|c|c|c|c|}
\hline \multirow[t]{2}{*}{ Genes } & \multicolumn{2}{|c|}{$\begin{array}{c}\text { Gene expression } \\
\log 2(\mathrm{TPM}+1) \text { for log-scale. }\end{array}$} & \multirow[t]{2}{*}{ Prognose } & \multirow[t]{2}{*}{ Correlation } \\
\hline & Normal & Tumor & & \\
\hline GPKOW & 4.2 & 4.5 & Favorable & 0.79 \\
\hline CCDC22 & 3.8 & 2.4 & Favorable & 0.76 \\
\hline WDR45" & 6.3 & 5.6 & Favorable & 0.65 \\
\hline WDR13 $^{\#}$ & 7.1 & 6.5 & Favorable & 0.38 \\
\hline ARAF $^{\#}$ & 5.7 & 5.6 & Favorable & 0.48 \\
\hline GRIPAP1\# & 5.3 & 5.0 & Favorable & 0.84 \\
\hline UXT & 7.1 & 7.3 & Favorable & 0.54 \\
\hline RBM10" & 5.2 & 5.2 & Favorable & 0.73 \\
\hline TSR2 & 5.5 & 5,5 & Unfavorable & 0.73 \\
\hline TIMM17B & 5.4 & 6.2 & Favorable & 0.57 \\
\hline PQBP1 ${ }^{\#}$ & 6.5 & 6.4 & Favorable & 0.51 \\
\hline NDUFB11 & 6.0 & 7.1 & Favorable & 0.40 \\
\hline HSD17B10 & 6.2 & 7.0 & Favorable & 0.51 \\
\hline TBC1D25" & 3.4 & 3.1 & Favorable & 0.70 \\
\hline SUV39H1 & 2.2 & 3.7 & Favorable & 0.67 \\
\hline FTSJ1 & 4.9 & 5.7 & Favorable & 0.50 \\
\hline PHKA2 ${ }^{\#}$ & 4.7 & 3.8 & Favorable & 0.65 \\
\hline SPIN2B ${ }^{\#}$ & 3.7 & 2.9 & $\mathrm{~N} / \mathrm{A}$ & 0.51 \\
\hline USP11 & 6.4 & 5.0 & Favorable & 0.72 \\
\hline IGBP1 $^{\#}$ & 6.0 & 5.4 & $\mathrm{~N} / \mathrm{A}$ & 0.50 \\
\hline APEX2 & 3.3 & 4.5 & Favorable & 0.58 \\
\hline LAS1L & 5.1 & 5.0 & Favorable & 0.51 \\
\hline RNF113A & 3.6 & 4.0 & Favorable & 0.45 \\
\hline FAM104B & 4.1 & 4.3 & Favorable & 0.45 \\
\hline TFE3 & 5.6 & 4.6 & Favorable & 0.70 \\
\hline TSPYL2 & 7.5 & 3.7 & Unfavorable & 0.63 \\
\hline ELK1 & 4.0 & 4.5 & N/A & 0.71 \\
\hline PRAF2 & 5.5 & 4.5 & Favorable & 0.48 \\
\hline IDH3G & 5.9 & 5.9 & Favorable & 0.41 \\
\hline MAGED2 ${ }^{\#}$ & 7.5 & 6.2 & $\mathrm{~N} / \mathrm{A}$ & 0.60 \\
\hline HDAC6" & 6.4 & 5.1 & Favorable & 0.58 \\
\hline PDHA1 & 6.6 & 7.0 & Favorable & 0.49 \\
\hline CDK16 & 5.7 & 6.4 & Favorable & 0.47 \\
\hline LOC550643 & 4.8 & 5.0 & N/A & \\
\hline SLC25A14 & 3.8 & 3.6 & Favorable & 0.55 \\
\hline PIN4 & 4.2 & 4.7 & $\mathrm{~N} / \mathrm{A}$ & 0.39 \\
\hline
\end{tabular}




\begin{tabular}{lllll} 
EBP & 4.6 & 7.3 & Favorable & 0.36 \\
LOC401588 & 2.5 & 1.4 & Favorable & 0.59 \\
USP27X $^{\#}$ & 2.0 & 1.6 & Favorable & 0.66 \\
SPSB3 $^{\text {CXorf40A }}$ & 6.2 & 5.4 & Favorable & 0.42 \\
C17orf59 & 4.0 & 4.2 & Favorable & 0.41 \\
SLC35A2 & 2.8 & 2.6 & Favorable & 0.35 \\
FAM156A & 4.0 & 4.8 & N/A & 0.63 \\
GABARAP\# & 4.6 & 3.3 & Favorable & 0.53 \\
NKAP & 9.7 & 9.0 & Favorable & 0.39 \\
RBMX2 & 2.8 & 3.0 & Favorable & 0.49 \\
UBA1 & 4.7 & 4.5 & Favorable & 0.43 \\
MSL3 $^{\#}$ & 7.1 & 7.3 & N/A & 0.60 \\
\hline
\end{tabular}


Table 2 (on next page)

Overall survival of miRNA that target OTUD5 in CESC. 


\begin{tabular}{|c|c|c|c|c|c|c|c|}
\hline miRNA & Low expression & High expression & Prognose & P value & HR & & \\
\hline Has-mir-584 & $\mathrm{n}=22$ & $\mathrm{n}=185$ & $\mathrm{~N} / \mathrm{A}$ & 0.1900 & $\begin{array}{l}1.39 \\
2.29)\end{array}$ & $(0.85$ & - \\
\hline Has-mir-137 & $n=156$ & $n=151$ & Unfavorable & 0.0280 & $\begin{array}{l}1.68 \\
2.69)\end{array}$ & $(1.05$ & - \\
\hline Has-mir-1293 & $\mathrm{n}=83$ & $n=224$ & $\mathrm{~N} / \mathrm{A}$ & 0.1800 & $\begin{array}{l}0.71 \\
1.18\end{array}$ & $(0.43$ & - \\
\hline Has-mir-1913 & $n=227$ & $\mathrm{n}=80$ & Unfavorable & 0.0210 & $\begin{array}{l}1.78 \\
2.93)\end{array}$ & $(1.09$ & - \\
\hline Has-mir-937 & $n=129$ & $\mathrm{n}=178$ & Unfavorable & 0.0023 & $\begin{array}{l}2.28 \\
3.94)\end{array}$ & $(1.32$ & - \\
\hline Has-mir-9-3 & $\mathrm{n}=76$ & $n=231$ & Favorable & 0.0310 & $\begin{array}{l}0.58 \\
0.96)\end{array}$ & $(0.35$ & - \\
\hline Has-mir-607 & $n=209$ & $n=98$ & Unfavorable & 0.0089 & $\begin{array}{l}1.86 \\
2.99)\end{array}$ & (1.16 & - \\
\hline Has-mir-3149 & $n=228$ & $\mathrm{n}=79$ & Unfavorable & 0.0054 & $\begin{array}{l}2.00( \\
3.29)\end{array}$ & & - \\
\hline Has-mir-144 & $\mathrm{n}=81$ & $n=226$ & Unfavorable & 0.0140 & $\begin{array}{l}2.09 \\
3.81)\end{array}$ & $(1.14$ & - \\
\hline Has-mir-103-1 & $n=159$ & $n=148$ & $\mathrm{~N} / \mathrm{A}$ & 0.0420 & $\begin{array}{l}1.62 \\
2.61)\end{array}$ & $(1.01$ & - \\
\hline
\end{tabular}




\section{Table 3 (on next page)}

Transcription factor of OTUD5 in QIAGEN, ConTra v3 and PROMO. 


\begin{tabular}{llllllll}
\hline Database & Gene & Correlation & P value & Database & Gene & Correlation & P value \\
\hline QIAGEN & EST1 & 0.12 & $\mathbf{0 . 0 3 8}$ & PROMO & IRF-2 & 0.33 & $\mathbf{2 . 7 e - 0 9}$ \\
& NKX2-5 & -0.07 & $\mathbf{0 . 2 5 0}$ & & RXRA & 0.10 & $\mathbf{0 . 0 8 4}$ \\
ConTra v3 & PAX4 & -0.02 & $\mathbf{0 . 6 8 0}$ & YY1 & 0.21 & $\mathbf{0 . 0 0 1}$ \\
& PPARG & 0.07 & $\mathbf{0 . 2 5 0}$ & XBP1 & 0.13 & $\mathbf{0 . 0 2 1}$ \\
& SP1 & 0.34 & $\mathbf{6 . 2 e - 1 0}$ & Cebpb & -0.04 & $\mathbf{0 . 4 8 0}$ \\
& SP2 & 0.40 & $\mathbf{7 . 1 e - 1 3}$ & PAX5 & 0.02 & $\mathbf{0 . 7 2 0}$ \\
SP3 & 0.26 & $\mathbf{6 . 1 e - 0 6}$ & TFIID & 0.21 & $\mathbf{0 . 0 0 1}$ \\
& SP4 & 0.32 & $\mathbf{7 . 6 e - 0 9}$ & FOXP3 & 0.27 & $\mathbf{1 . 9 e - 0 6}$ \\
& MAZ\# & 0.23 & $\mathbf{4 . 0 e - 0 5}$ & GR & 0.12 & $\mathbf{0 . 0 3 9}$ \\
& MAZR & 0.37 & $\mathbf{1 . 9 e - 1 1}$ & GTF2I & 0.31 & $\mathbf{4 . 7 e - 0 8}$ \\
& KLF4 & 0.02 & $\mathbf{0 . 7 6 0}$ & NF-1 & 0.32 & $\mathbf{9 . 1 e - 0 9}$ \\
& KLF5 & 0.06 & $\mathbf{0 . 3 0 0}$ & ESR1 & 0.13 & $\mathbf{0 . 0 1 9}$ \\
& KLF16 & -0.02 & $\mathbf{0 . 7 2 0}$ & SLEB11 & -0.02 & $\mathbf{0 . 7 8 0}$ \\
& TCN1 & -0.11 & $\mathbf{0 . 0 5 8}$ & ETS1 & 0.12 & $\mathbf{0 . 0 3 8}$ \\
& ELF4 & 0.28 & $\mathbf{4 . 2 e - 0 7}$ & ELK1\# & 0.71 & $\mathbf{2 . 2 e - 4 7}$ \\
& & & TFAP2A & 0.10 & $\mathbf{0 . 1 0 0}$ \\
& & & SP1 & 0.34 & $\mathbf{0 . 0 3 8}$ \\
& & & HMGA1 & 0.10 & $\mathbf{0 . 1 3 0}$ \\
& & & P53 & 0.37 & $\mathbf{2 . 6 e - 1 1}$ \\
\hline
\end{tabular}


Table 4 (on next page)

Kinase of OTUD5 in CESC from LinkedOmics. 


\begin{tabular}{lllll} 
Gene & size & FDR & \multicolumn{2}{l}{$\begin{array}{l}\text { Gene expression log2(TPM + 1) } \\
\text { for log-scale. }\end{array}$} \\
\hline & & & Normal & Tumor \\
PLK1 & 38 & 0.0075271 & 1.6 & 5.3 \\
ATM & 50 & 0.015054 & 4.3 & 2.7 \\
PIK3CA & 4 & 0.067242 & 3.2 & 3.2 \\
CDK5 & 28 & 0.074669 & 3 & 4.2 \\
CHEK1 & 46 & 0.078282 & 1.4 & 4.2 \\
\hline
\end{tabular}

1

2 
Table 5 (on next page)

Ubiquitin ligase of OTUD5 in CESC from UbiBrowser. 


\begin{tabular}{|c|c|c|c|c|c|c|c|}
\hline \multirow[t]{2}{*}{ Genes } & \multirow[t]{2}{*}{ SCORE } & \multicolumn{2}{|c|}{$\begin{array}{l}\text { Gene expression } \\
\log 2(\mathrm{TPM}+1) \text { for } \\
\text { log-scale. }\end{array}$} & \multirow[t]{2}{*}{ Genes } & \multirow[t]{2}{*}{ SCORE } & \multicolumn{2}{|c|}{$\begin{array}{c}\text { Gene expression } \\
\log 2(\mathrm{TPM}+1) \\
\text { for log-scale. }\end{array}$} \\
\hline & & Normal & Tumor & & & Normal & Tumor \\
\hline RNF180 & 0.719 & 1.9 & 0.1 & FBXO3 & 0.623 & 4.6 & 4.0 \\
\hline NEDD4L $^{\#}$ & 0.705 & 3.0 & 4.7 & HERC2 & 0.623 & 5.1 & 4.8 \\
\hline RNF6 $^{\#}$ & 0.694 & 3.4 & 3.9 & TRIM11 & 0.610 & 3.8 & 4.6 \\
\hline MARCH1 & 0.694 & 1.8 & 1.3 & KLHL13 & 0.610 & 4.1 & 1.9 \\
\hline MDM2 ${ }^{\#}$ & 0.668 & 5.0 & 6.0 & MARCH8 & 0.610 & 3.6 & 2.8 \\
\hline SYVN1 & 0.667 & 5.3 & 4.8 & RFPL4A & 0.610 & - & - \\
\hline PIAS4 & 0.648 & 3.7 & 3.8 & MARCH7 & 0.608 & 5.2 & 5.3 \\
\hline MIB1 & 0.642 & 3.4 & 2.8 & RNF216 & 0.608 & 4.1 & 3.4 \\
\hline ZC3HC1 & 0.623 & 3.7 & 3.6 & MIB2 & 0.594 & 6.1 & 5.1 \\
\hline & & & & UBE3C & 0.594 & 4.6 & 4.6 \\
\hline
\end{tabular}

1 